TRANSACTIONS OF THE

AMERICAN MATHEMATICAL SOCIETY

Volume 355, Number 1, Pages 119-142

S 0002-9947(02)03111-2

Article electronically published on September 5, 2002

\title{
A HIGHER LEFSCHETZ FORMULA FOR FLAT BUNDLES
}

\author{
MOULAY-TAHAR BENAMEUR
}

\begin{abstract}
In this paper, we prove a fixed point formula for flat bundles. To this end, we use cyclic cocycles which are constructed out of closed invariant currents. We show that such cyclic cocycles are equivariant with respect to isometric longitudinal actions of compact Lie groups. This enables us to prove fixed point formulae in the cyclic homology of the smooth convolution algebra of the foliation.
\end{abstract}

\section{INTRODUCTION}

Index theory has gained ground during the last ten years and the appearance of the non-commutative-geometry point of view has opened up the way to encompass new singular spaces [16, 18, 19, 21, 34, 12, 11, 32, 27, 31, 8. Among these spaces, the space of leaves of a compact foliated manifold plays a central role and appears in a decisive way in the understanding of innumerable problems; see, for instance, 22] and also [10].

For foliations, the index theorem has been proved by Connes and Skandalis in [13] and [20]. In this last paper, the authors proved that the index of a longitudinal elliptic pseudodifferential operator on a compact foliated manifold is an analytic class that lives in the $K$-theory of the $C^{*}$-algebra of the foliation. Moreover, they computed this class in topological terms by using shrieck maps between spaces of leaves. The index formula obtained in this way thus takes place more precisely in the $K$-theory group of the smooth convolution algebra of the holonomy groupoid of the foliation. In order to deduce scalar index formulae, one is then led to the definition of the Chern character for noncommutative algebras [17]. More precisely, Connes has succeeded in defining the cyclic cohomology as a counterpart for de Rham homology, and he proved its pairing with $K$-theory. This pairing then enabled him to go further and to prove scalar universal index formulae for longitudinal elliptic operators in the presence of cyclic cocycles on the smooth convolution algebra of the holonomy groupoid, see [16, 15].

For general foliations, such formulae are very deep and remain at present far from understood. On the other hand, the recent discoveries concerning the link between the measured index theorem for flat bundles and the Atiyah-Patodi-Singer eta invariant [3], proved by Douglas, Hurder and Kaminker in their fantastic paper 22], have reinvigorated the index theory of flat bundles. It then became urgent to investigate more systematically the Connes cyclic index formula in this first

Received by the editors November 23, 2001 and, in revised form, March 12, 2002.

2000 Mathematics Subject Classification. Primary 19L47, 19M05, 19K56.

Key words and phrases. $C^{*}$-algebra, K-theory, Lefschetz, foliations. 
enlightening example. The computations in this case were carried out by Nistor in [32] and more recently by Jiang in [27.

From the beginning of the index theory, the equivariant versions of many index formulae gave interesting applications to fixed point formulae or to rigidity with respect to compact Lie group actions, and also produced fruitful integrality results 5, 4, 2, 35, 24, 25, 6, 7. For foliations for instance, one obtains equivariant versions of the Connes-Skandalis index theorem $[6]$ and also equivariant versions of the measured index theorem [14, 24]. The goal of the present paper is to prove a higher cyclic fixed point formula for flat bundles, by using higher cyclic cocycles on the smooth convolution algebra of the horizontal foliation.

Let $M$ be a smooth compact manifold and denote by $\Gamma$ the fundamental group of $M$. Assume that there exists a locally free action $\varrho$ of $\Gamma$ by diffeomorphisms on a smooth compact manifold $\mathcal{T}$. Then we can define the suspension $V=\tilde{M} \times_{\Gamma} \mathcal{T}$ of this action, which is the total space of a flat bundle $V \rightarrow M$ over $M$. The flat connection enables us to endow $V$ with a smooth foliation $F$. Assume now that $\tilde{g}$ is an isometry of $\tilde{M} \times \mathcal{T}$ with respect to a $\Gamma$-invariant metric, which is the lift of some leaf-preserving isometry $g$ of $V$. The first problem that we solve here is the definition of the Lefschetz number of $\tilde{g}$ with respect to any elliptic $\Gamma$-invariant longitudinal operator and any $\Gamma$-invariant current on $\mathcal{T}$. This result generalizes the previous ones obtained in the nonequivariant case [27, and we recover the Connes cyclic index map evoked above when $g$ is the identity map [16]. The computation of the Lefschetz number in terms of topological data over the fixed point manifold in the spirit of [5] 4] is then obtained by using Haefliger's integration along the leaves together with the $K$-theory Lefschetz theorem as proved in [6]. The formula thus obtained gives information on foliated Lefschetz numbers for noncompact manifolds that $\Gamma$-cover compact ones; this is in the spirit of [1].

Let us describe now more precisely the results of the present paper. Given a $\Gamma$-invariant current $C$ and using Haefliger's integration along the leaves [23], we construct a cyclic cocycle $\tau_{C}$ over the smooth convolution algebra $C_{c}^{\infty}(\mathcal{G})$ of the holonomy groupoid $\mathcal{G}$ of $(V, F)$. Denote by $G$ the compact Lie group generated by $g$. A very simple remark on the existence of a $G$-equivariant representative of $\tau_{C}$ enables us to prove a pairing between the de Rham homology class of $C$ and the $G$-equivariant $K$-theory group of the smooth algebra $C_{c}^{\infty}(\mathcal{G})$. In the example of a $\Gamma$-invariant measure on $\mathcal{T}$, one gets in this way the well-known pairing that defines the measured Lefschetz number of $g$; see [24] and also [6]. For higher $\Gamma$-invariant currents, the results are new and yield to a definition of the $C$-Lefschetz number of $\tilde{g}$ with respect to any elliptic $G$-invariant longitudinal $\Gamma$-invariant pseudodifferential operator. The next step is then to express this $C$-Lefschetz number in terms of topological data over the fixed point submanifold of $G$ with its induced foliation. This is carried out in the last section and we obtain, for instance, the following theorem; see the usual notation in Section 4 and Theorem 7

Theorem 1. The C-Lefschetz number of the isometry $g$ with respect to the longitudinally elliptic pseudodifferential $G$-invariant operator $P$ is given by the following fixed point topological formula:

$$
\mathbb{L}_{C}(g ; P)=\left\langle\phi^{V^{G}}\left(\pi_{F^{G}} ! \frac{\operatorname{Ch}\left(i^{*}[\sigma(P)]\right)(g)}{\operatorname{Ch}\left(\lambda_{-1}\left(N^{G} \otimes \mathbb{C}\right)(g)\right)} p^{*} \operatorname{Td}\left(p_{0}\left(B^{G}\right)\right)\right),\left.C\right|_{A^{G}}\right\rangle,
$$


where $p_{0}\left(B^{G}\right)$ is the projection in $M$ of the fixed point submanifold $V^{G}$ of $G$, and $N^{G}$ is the normal vector bundle to $V^{G}$ in $V$.

As a simple consequence, we immediately obtain an interesting integrality result, which needs to be expanded elsewhere, i.e., when $C$ is itself the Chern-Connes character of a $\Gamma$-invariant elliptic operator on $\mathcal{T}$, the characteristic number in the right-hand side of the Lefschetz formula belongs to the subgroup $R(G)(g)$ of $\mathbb{C}$ composed of evaluations at $g$ of characters of $G$. In particular, we get an integer when $g$ is an involution.

Acknowledgements. I wish to thank V. Nistor for several helpful discussions. Part of this work was done during a visit to the Geometric Functional Analysis group at Penn State University, and I wish to thank all the members for their warm hospitality. Finally, I am indebted to the referee for helping considerably to improve the presentation of this paper and also for pointing out some mistakes in the first version.

\section{Review of the $K$-THEORY LefschetZ THEOREM}

We shall recall in this first section some basic results pertaining to the equivariant index theory for flat bundles. Our references are mainly [20, 16] and, for the equivariant case, [6. Since we shall only need the longitudinal index theorem for flat bundles or transverse closed submanifolds of such bundles, we restrict ourselves to this geometric situation.

Let $M$ be a smooth compact manifold and let $\Gamma$ be the fundamental group of $M$. We denote by $\tilde{M}$ the universal $\Gamma$-cover of $M$. Let $\mathcal{T}$ be a smooth compact manifold, and assume that we are given a (locally free) smooth action $\varrho: \Gamma \rightarrow \operatorname{Diff}(\mathcal{T})$ of $\Gamma$ in the diffeomorphism group of the manifold $\mathcal{T}$. Then we consider the smooth compact manifold $V:=\tilde{M} \times_{\Gamma} \mathcal{T}$ which is the quotient of the product manifold $\tilde{M} \times \mathcal{T}$ by the free and proper action of $\Gamma$ on the right given for $\gamma \in \Gamma$ and for any $(\tilde{m}, t) \in \tilde{M} \times \mathcal{T}$ by

$$
(\tilde{m}, t) \gamma:=\left(\tilde{m} \gamma, \varrho\left(\gamma^{-1}\right)(t)\right) .
$$

The smooth manifold $V$ then fibres over the smooth manifold $M$ so that the typical fibres are copies of the manifold $\mathcal{T}$. We thus have a commutative diagram of fibrations:

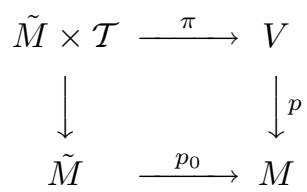

where $\pi$ and $p_{0}$ are quotient projections with respect to the action of $\Gamma$ and $p$ is the fibration projection induced by the first component projection on the left of the diagram. In addition, we have a well-defined smooth foliation on the compact manifold $V$ with leaves given by the projections under $\pi$ of the submanifolds $\tilde{M} \times\{t\}$ when $t$ runs through $\mathcal{T}$. We shall denote by $F$ the longitudinal bundle of this foliation, i.e., the tangent vector bundle to the leaves. The total space of this bundle is more precisely given by

$$
F:=(T \tilde{M} \times \mathcal{T}) / \Gamma
$$

where $T \tilde{M}$ is the $\Gamma$-equivariant tangent vector bundle to the manifold $\tilde{M}$. Here the action of $\Gamma$ on $T \tilde{M} \times \mathcal{T}$ is the diagonal one. The leaves of this foliation are 
transverse to the fibres of the fibration $p: V \rightarrow M$ and we get in this way a foliated bundle $(V, F, M)$.

We denote by $\mathcal{G}$ the smooth groupoid:

$$
\mathcal{G}:=(\tilde{M} \times \tilde{M} \times \mathcal{T}) / \Gamma \quad \text { with space of units } \mathcal{G}^{(0)}=V,
$$

where $\Gamma$ acts again diagonally and where the source and range maps of $\mathcal{G}$ are defined by

$$
s\left[\tilde{m}, \tilde{m}^{\prime}, t\right]:=\left[\tilde{m}^{\prime}, t\right] \in V \quad \text { and } \quad r\left[\tilde{m}, \tilde{m}^{\prime}, t\right]:=[\tilde{m}, t] \in V .
$$

We shall denote by $\nu$ the transverse vector bundle of $(V, F)$ defined as the quotient vector bundle $\nu:=T V / F$. Let $T_{\text {vert }} V$ be the vertical tangent bundle of the fibration $p: V \rightarrow M$, i.e.,

$$
T_{\text {vert }} V:=\operatorname{Ker}\left(p_{*}\right) \subset T V .
$$

Then $T_{\text {vert }} V$ is a supplementary vector bundle of the longitudinal bundle $F$ and is therefore isomorphic to $\nu$. The total space of $T_{\text {vert }} V$ can also be defined as the quotient manifold of $\tilde{M} \times T \mathcal{T}$ by the diagonal action of $\Gamma$. The action of the group $\Gamma$ on $T(\mathcal{T})$ is as usual by the differentials of $\varrho$. We assume for simplicity in the whole paper that the manifold $\tilde{M}$ is orientable and oriented, so that the foliation $(V, F)$ is oriented.

Let $E$ be a smooth $\mathbb{Z}_{2}$-graded vector bundle over $V$ and denote by $\tilde{E}$ the pullback of $E$ to $\tilde{M} \times \mathcal{T}$. So $\tilde{E}$ is a $\Gamma$-equivariant vector bundle over the $\Gamma$-manifold $\tilde{M} \times \mathcal{T}$. The restriction of $\tilde{E}$ to the subspaces $\tilde{M} \times\{t\}, t \in \mathcal{T}$ enables us to define $\psi^{m}(\tilde{M} \times \mathcal{T}, \tilde{E})$ as the space of smooth families $\left(P_{t}\right)_{t \in \mathcal{T}}$ of classical pseudodifferential operators on $\tilde{M}$, of order $m \in \mathbb{Z}$, with coefficients in $\tilde{E}$. Hence the union

$$
\psi^{\infty}(\tilde{M} \times \mathcal{T}, \tilde{E}):=\bigcup_{m \in \mathbb{Z}} \psi^{m}(\tilde{M} \times \mathcal{T}, \tilde{E})
$$

is a filtered algebra for the composition of pseudodifferential operators over $\tilde{M}$ with coefficients in $\tilde{E}$. This algebra fibres smoothly over the compact manifold $\mathcal{T}$ (the space $\psi^{\infty}(\tilde{M} \times \mathcal{T}, \tilde{E})$ being endowed as is customary with the topology of kernels, see [33, 29]). The group $\Gamma$ then acts naturally on $\psi^{\infty}(\tilde{M} \times \mathcal{T}, \tilde{E})$ by algebra automorphisms.

Definition 1. A longitudinal pseudodifferential operator of order $m$ on $(V, F)$ with coefficients in $E$ is a smooth section

$$
P: \mathcal{T} \rightarrow \psi^{m}(\tilde{M} \times \mathcal{T}, \tilde{E}),
$$

such that for any $\gamma \in \Gamma$,

$$
P_{\varrho(\gamma)(t)}=\gamma \cdot P_{t}, \quad \forall \gamma \in \Gamma .
$$

where $\gamma \cdot P_{t}$ is the operator obtained from $P_{t}$ by conjugation, using the actions of $\Gamma$ on $\tilde{M} \times \mathcal{T}$ and $\tilde{E}$.

We shall call such an operator a $\Gamma$-invariant operator for short. So for any $t \in \mathcal{T}$, the operator $P_{t}$ is a pseudodifferential operator of order $m \in \mathbb{Z}$ with coefficients in $\left.\tilde{E}\right|_{\tilde{M} \times\{t\}}$. The principal symbol $\sigma(P)$ of the longitudinal pseudodifferential operator $P$ is defined as the smooth map $t \mapsto \sigma\left(P_{t}\right)$ that assigns to $t \in \mathcal{T}$ the principal symbol of the pseudodifferential operator $P_{t}$. So $\sigma(P)$ is a section of the bundle $\operatorname{End}\left(\pi^{*} \tilde{E}\right)$ 
over $\mathcal{T} \times T^{*} \tilde{M} \backslash \tilde{M}$, where $\pi: \mathcal{T} \times T^{*} \tilde{M} \backslash \tilde{M} \rightarrow \mathcal{T} \times \tilde{M}$ is the projection. The section $\sigma(P)$ is, in addition, smooth and positively $m$-homogeneous, i.e.,

$$
\sigma\left(P_{t}\right)(\tilde{m}, \lambda \xi)=\lambda^{m} \sigma\left(P_{t}\right)(\tilde{m}, \xi), \quad \forall \lambda>0 .
$$

A longitudinal pseudodifferential operator $P$ on $(V, F)$ is elliptic if, for any $t \in \mathcal{T}$, the operator $P_{t}$ is elliptic, i.e., if for any $t \in \mathcal{T}$ the principal symbol $\sigma\left(P_{t}\right)(\tilde{m}, \xi)$ of $P_{t}$ is an automorphism of $\tilde{E}_{\tilde{m}, t}$ for any $(\tilde{m}, \xi) \in T^{*} \tilde{M} \backslash \tilde{M}$.

By the Schwartz theorem, we can associate to any longitudinal pseudodifferential operator $P$ a distributional kernel that is a smooth family $\left(k_{t}\right)_{t \in \mathcal{T}}$, where $k_{t}$ is the Schwartz kernel of $P_{t}$ acting over $\tilde{M}$. Since $P$ is $\Gamma$-invariant in the sense of Equation (II), this Schwartz kernel yields a distributional section $k$ over the groupoid $\mathcal{G}$, well defined by the equality:

$$
k\left[\tilde{m}, \tilde{m}^{\prime}, t\right]:=k_{t}\left(\tilde{m}, \tilde{m}^{\prime}\right) .
$$

The pseudodifferential operator $P$ will be called a compactly supported pseudodifferential operator if the distributional kernel of $P$ is compactly supported as a distributional section over $\mathcal{G}$. We denote by $\psi^{m}(V, F ; E)$ the space of compactly supported longitudinal pseudodifferential operators of order $m \in \mathbb{Z}$, on $(V, F)$, with coefficients in $E$. The ideal $\psi^{-\infty}(V, F ; E)$ of regularizing operators in $\psi^{\infty}(V, F ; E)$ is defined by

$$
\psi^{-\infty}(V, F ; E):=\bigcap_{m \in \mathbb{Z}} \psi^{m}(V, F ; E) .
$$

This ideal can be identified under the Schwartz isomorphism, with the smooth convolution algebra $C_{c}^{\infty}(\mathcal{G}, \operatorname{End}(E))$ of the groupoid $\mathcal{G}$, see 33. Hence we have an exact sequence:

$$
0 \rightarrow C_{c}^{\infty}(\mathcal{G}, \operatorname{End}(E)) \hookrightarrow \psi^{\infty}(V, F ; E) \longrightarrow \mathcal{A}(V, F ; E) \rightarrow 0,
$$

where $\mathcal{A}(V, F ; E)$ is the quotient algebra of longitudinal complete symbols. The parametrix construction in the smooth manifold $\tilde{M}$ easily shows that if $P \in$ $\psi^{\infty}(V, F ; E)$ is elliptic, then its class in $\mathcal{A}(V, F ; E)$ is invertible and defines a homotopy class in $K_{1}(\mathcal{A}(V, F ; E))$ that coincides with the class $[\sigma(P)]$ of the principal symbol. See also [14.

Definition 2. The index $\operatorname{Ind}(P)$ of the longitudinal elliptic pseudodifferential operator $P$ is defined as the image under the boundary map

$$
\partial: K_{1}(\mathcal{A}(V, F ; E)) \longrightarrow K_{0}\left(C_{c}^{\infty}(\mathcal{G}, \operatorname{End}(E))\right) \simeq K_{0}\left(C_{c}^{\infty}(\mathcal{G})\right),
$$

of the homotopy class of the principal symbol.

In the above definition, we have used the Morita equivalence between the two algebras $C_{c}^{\infty}(\mathcal{G}, \operatorname{End}(E))$ and $C_{c}^{\infty}(\mathcal{G})$ to view the index class $\operatorname{Ind}(P)$ as an element of the $K$-theory of the smooth convolution algebra $C_{c}^{\infty}(\mathcal{G})$. We also point out that any fibre of the fibration $V \rightarrow M$ is a complete transversal for the foliation $(V, F)$. Therefore, the algebra $C_{c}^{\infty}(\mathcal{G})$ is also Morita equivalent to the crossed product algebra $C^{\infty}(\mathcal{T}) \rtimes_{\varrho} \Gamma$. The image of the index of $P$ in the $K$-theory of the completion $C^{*}$-algebra $C(\mathcal{T}) \rtimes \Gamma$ can be computed by using Kasparov's theory, see 10.

Let now $\tilde{g}$ be an isometry of $\tilde{M} \times \mathcal{T}$ for some $\Gamma$-invariant Riemannian metric on $\tilde{M} \times \mathcal{T}$, which preserves the longitudinal bundle $T \tilde{M} \times \mathcal{T}$ over $\tilde{M} \times \mathcal{T}$ and induces an isometry $g$ of $V$. More precisely, we fix an automorphism $\beta$ of $\Gamma$ so that the action of $\tilde{g}$ is equivariant up to the shift by $\beta$. We denote by $G$ the compact 
Lie group generated by $g$ in the group of isometries of $V$ for the induced metric. The compact Lie group $G$ then acts on the foliated bundle $(V, F)$ by $F$-preserving isometries. Therefore, $G$ sends each leaf of $(V, F)$ to a leaf of $(V, F)$. The action of $\tilde{g}$ induces a diffeomorphism $\alpha(g)$ on the compact manifold $\mathcal{T}$. Moreover, we are given, for any $t \in \mathcal{T}$, a diffeomorphism $g(t)$ of $\tilde{M}$, so that the induced action of $g$ on $V$ can be written as:

$$
g[\tilde{m}, t]:=[g(t)(\tilde{m}), \alpha(g)(t)], \quad \forall[\tilde{m}, t] \in V .
$$

Therefore, we have the following equivariance relations:

$$
\begin{array}{r}
\alpha(g) \circ \varrho(\gamma)=(\varrho \circ \beta)(\gamma) \circ \alpha(g) \text { and } g\left(\varrho\left(\gamma^{-1}\right)(t)\right)(\tilde{m} \gamma)=g(t)(\tilde{m}) \beta(\gamma), \\
\forall(\tilde{m}, t, \gamma) \in \tilde{M} \times \mathcal{T} \times \Gamma .
\end{array}
$$

The simplest example of such a diffeomorphism $\tilde{g}$ of $\tilde{M} \times \mathcal{T}$ is when $\beta$ is the identity automorphism of $\Gamma$, say when $\tilde{g}$ is equivariant for the action of $\Gamma$. In this case, the equivariance relations become

$$
\begin{aligned}
\alpha(g) \circ \varrho(\gamma)=\varrho(\gamma) \circ \alpha(g) \text { and } g\left(\varrho\left(\gamma^{-1}\right)(t)\right)(\tilde{m} \gamma)= & g(t)(\tilde{m}) \gamma, \\
& \forall(\tilde{m}, t, \gamma) \in \tilde{M} \times \mathcal{T} \times \Gamma .
\end{aligned}
$$

In practice, it is often true that an isometry of $V$ can be induced by a $\Gamma$-equivariant isometry of $\tilde{M} \times \mathcal{T}$, but not always. What can be insured is the $\beta$-equivariance relations (2) for some automorphism $\beta$ of $\Gamma$. We point out that only exterior automorphisms are consistent in this discussion. More precisely, when $\beta$ is an interior automorphism, one can modify the map $t \mapsto g(t)$ to get the allowed equivariance property (3). This is true, for instance, when the isometry $g$ is assumed to preserve the leaves of $(V, F)$, see Section 3 .

In the above discussion, we have dealt with a single isometry $g$, but once the lift $\tilde{g}$ of $g$ is fixed, the lifts of the powers of $g$ are defined to be the powers of $\tilde{g}$.

Remark 1 . In the more general case of an action of a not necessarily cyclic group $H$, the choice of a lift of the action can be done by fixing a homomorphism $\beta: H \rightarrow$ $\operatorname{Aut}(\Gamma)$, say an action of $H$ on $\Gamma$. This lifted action can be taken to be $\Gamma$-equivariant if $\beta(H)$ is a subgroup of the group $\operatorname{Int}(\Gamma)$ of interior automorphisms of $\Gamma$.

Let $E$ be a $G$-equivariant $\mathbb{Z}_{2}$-graded vector bundle over $V$. A longitudinal pseudodifferential operator $P \in \psi^{\infty}(V, F ; E)$ is $G$-invariant if we have $g . P=P$. More precisely, if $t \in \mathcal{T}$ and $\xi \in C_{c}^{\infty}(\tilde{M}, \tilde{E})$, then $g . P$ is defined by

$$
(g \cdot P)(t)(\xi)(\tilde{m}):=\tilde{g}\left[P_{\alpha(g)^{-1}(t)}\left(g^{-1} \xi\right)\left(g^{-1}(t)(\tilde{m})\right)\right],
$$

where $P_{\alpha(g)^{-1}(t)}\left(g^{-1} \xi\right)\left(g^{-1}(t)(\tilde{m})\right) \in \tilde{E}_{\left(g^{-1}(t)(\tilde{m}), \alpha\left(g^{-1}\right)(t)\right)}$ and where we have used the action of $\tilde{g}$ on $\tilde{E}$ to get an element of $\tilde{E}_{(\tilde{m}, t)}$. Note that when $P$ is $\Gamma$-invariant in the sense of Equation (11), g.P is also $\Gamma$-invariant. In the same way, when the pseudodifferential operator is a regularizing operator $P$, so is $g . P$. In particular, we have a well-defined action of $G$ on the smooth groupoid $\mathcal{G}$ given more precisely by

$$
g\left[\tilde{m}, \tilde{m}^{\prime}, t\right]:=\left[g(t)(\tilde{m}), g(t)\left(\tilde{m}^{\prime}\right), \alpha(g)(t)\right] .
$$

The pseudodifferential exact sequence described above can be used by means of the boundary map in $G$-equivariant $K$-theory to associate to any longitudinally elliptic $G$-invariant pseudodifferential operator $P$ on $(V, F ; E)$ a $G$-equivariant index class 
$\operatorname{Ind}_{G}(P)$ in the group $K^{G}\left(C_{c}^{\infty}(\mathcal{G})\right)$ of equivariant $K$-theory, see [6]. The inclusion of the algebra $C_{c}^{\infty}(\mathcal{G})$ as a $G$-subalgebra of Connes' $C^{*}$-algebra $C^{*}(V, F)$ of the foliation [13] enables us to view the $G$-index as an equivariant $K$-theory class of $C^{*}(V, F)$. Note that the Haar system on $\mathcal{G}$ used here in the definition of the $C^{*}$ algebra $C^{*}(V, F)$ is simply given by a $\Gamma$-invariant Lebesgue measure fixed on $\tilde{M}$ and $G_{1}$-invariant, where $G_{1}$ is the dense subgroup of $G$ composed of powers of $g$. We also denote the index class of $P$ in $K^{G}\left(C^{*}(V, F)\right)$ by $\operatorname{Ind}_{G}(P)$ for simplicity.

We denote by $R(G)$ the representation ring of $G$. The ring $R(G)$ can also be described by using characters. Since the group $K^{G}\left(C^{*}(V, F)\right)$ is obviously endowed with the structure of an $R(G)$-module, we can localize it with respect to any prime ideal of $R(G)$. We shall concentrate on the prime ideal corresponding to the element $g$, say the set of characters that vanish at $g$, and we denote by $R(G)_{g}$ the ring of such fractions, we also denote, for instance, by $K^{G}\left(C^{*}(V, F)\right)_{g}$ the $R(G)_{g}$-module of fractions thus obtained.

Definition 3. Let $P$ be a $G$-invariant longitudinally elliptic pseudodifferential operator on $(V, F)$. The class of the $G$-index $\operatorname{Ind}_{G}(P)$ in the fraction module $K^{G}\left(C^{*}(V, F)\right)_{g}$ is called the Lefschetz class of $g$ with respect to $P$ and denoted by $\mathbb{L}(g ; P)$. So

$$
\mathbb{L}(g ; P):=\frac{\operatorname{Ind}_{G}(P)}{1_{R(G)}} \quad \in K^{G}\left(C^{*}(V, F)\right)_{g} .
$$

This definition is inspired from the usual definition of the Lefschetz class and the Lefschetz number for manifolds, see [5]. When the manifold $\mathcal{T}$ is reduced to a point, we obtain a Lefschetz class that belongs to the module $K^{G}\left(C_{c}^{\infty}\left(\tilde{M} \times_{\Gamma} \tilde{M}\right)\right)_{g}$; compare [16] page 233]. Morita equivalence enables us then to view the Lefschetz class of a $\Gamma$-equivariant isometry of the universal cover $\tilde{M}$ as an element of the tensor product module $K\left(C_{r e d}^{*} \Gamma\right) \otimes R(G)_{g}$, where $C_{r e d}^{*} \Gamma$ is the reduced $C^{*}$-algebra of the discrete group $\Gamma$. When $\mathcal{T}$ is not reduced to a point, our Lefschetz class takes into account the longitudinal and the transverse structure of the foliated manifold $(V, F)$.

Now let $\tilde{X}$ be the subset of $\tilde{M} \times \mathcal{T}$ of those couples $(\tilde{m}, t)$ that satisfy

$$
\exists \gamma \in \Gamma, \alpha(g)(t)=\varrho\left(\gamma^{-1}\right)(t) \text { and } g(t)(\tilde{m})=\tilde{m} \gamma .
$$

Then $\tilde{X}$ is a disjoint union over the conjugacy classes $[\gamma]$ of $\gamma$ of subsets $\tilde{X}_{[\gamma]}$ corresponding to elements $\alpha$ belonging to $[\gamma]$, and which are globally $\Gamma$-invariant. Note that the subspaces $\tilde{X}$ and $\tilde{X}_{[\gamma]}$ are smooth closed submanifolds of $\tilde{M} \times \mathcal{T}$, where $\tilde{X}_{[\gamma]}$ is, for instance, the inverse image under the projection of the fixed point submanifold $V^{G}$ of $G$ or $g$, acting on $V$. Assume as is customary that $V^{G}$ is transverse to the leaves of $F$ [25] and denote by $F^{G}$ the integrable vector bundle $F \cap T V^{G}$ over $V^{G}$. Let $N^{G}$ be the normal vector bundle to $V^{G}$ in $V$, with respect to the $G$-invariant metric fixed on $V$. Then the complex exterior powers $\Lambda^{i}\left(N^{G} \otimes \mathbb{C}\right)$ are complex $G$-equivariant vector bundles over $V^{G}$ and define $G$-equivariant $K$ theory classes. We denote by $\lambda_{t}\left(N^{G} \otimes \mathbb{C}\right)$ the formal class $\sum_{i} t^{i}\left[\Lambda^{i}\left(N^{G} \otimes \mathbb{C}\right)\right]$ and by $\lambda_{-1}\left(N^{G} \otimes \mathbb{C}\right)$ the corresponding class in $K_{G}\left(V^{G}\right) \simeq K\left(V^{G}\right) \otimes R(G)$.

The manifold $\tilde{X}$ is a foliated manifold that is endowed with a free and proper action of the discrete group $\Gamma$ and another action of the compact Lie group $G$. Recall that transversality means that the dimension of $\tilde{X}$ is $\geq \operatorname{dim}(\mathcal{T})$ and that for 
any $(\tilde{m}, t) \in \tilde{X}$, we have

$$
\operatorname{dim}\left(T_{(\tilde{m}, t)} \tilde{X} \cap T_{\tilde{m}} \tilde{M}\right)+\operatorname{dim}(\mathcal{T})=\operatorname{dim}\left(T_{(\tilde{m}, t)} \tilde{X}\right) .
$$

We denote by $A^{G}$ the projection of $\tilde{X}$ in $\mathcal{T}$. The transversality assumption then implies that the $\Gamma$-space $A^{G}$ is a clopen submanifold of $\mathcal{T}$. The leaves of the foliation of $\tilde{X}$ are the intersections of $\tilde{X}$ with the leaves $\tilde{M} \times\{t\}$ of $\tilde{M} \times \mathcal{T}$. Each connected component of $\tilde{X}$ is therefore endowed with a foliation $F_{\tilde{X}}$ of constant dimension. The quotient foliation of $F_{\tilde{X}}$ with respect to the action of $\Gamma$ is then exactly $\left(V^{G}, F^{G}\right)$.

We have again a commutative diagram of projections:

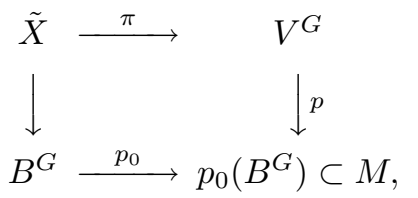

where $B^{G}$ is the projection of $\tilde{X}$ in $\tilde{M}$. Note that the normal vector bundle $N^{G}$ to $F^{G}$ is the quotient under the corresponding action of $\Gamma$ of a normal vector bundle $N_{\tilde{X}}$ to $F_{\tilde{X}}$ in $\tilde{X}$, with respect to the $(\Gamma, G)$-invariant metric on $\tilde{M} \times \mathcal{T}$. We shall denote by $\mathcal{G}_{X}$ the Lie groupoid

$$
\mathcal{G}_{X}:=\left[\bigcup_{t \in A^{G}}\left(\tilde{X}_{t} \times \tilde{X}_{t} \times\{t\}\right)\right] / \Gamma .
$$

Now, the space of pseudodifferential operators along the fibres of the fibration $\tilde{X} \rightarrow A^{G}$ with coefficients in a given $\Gamma$-equivariant $\mathbb{Z}_{2}$-graded vector bundle $\tilde{E}$ over $\tilde{X}$ is itself a well-defined $\Gamma$-equivariant bundle over $A^{G}$. A smooth section of this bundle, i.e., a family pseudodifferential operator $\tilde{P}:=\left(\tilde{P}_{t}\right)_{t \in A^{G}}$ on the fibration $\tilde{X} \rightarrow A^{G}$ that is elliptic along the fibres and $\Gamma$-invariant in the sense of Equation 1 is, by definition, a longitudinally elliptic pseudodifferential operator $P$ on the compact foliated manifold $\left(V^{G}, F^{G}\right)$. If we denote by $\psi^{\infty}\left(V^{G}, F^{G} ; E\right)$ the space of such family operators and by $\psi^{-\infty}\left(V^{G}, F^{G} ; E\right)$ the ideal $\bigcap_{m \in \mathbb{Z}} \psi^{m}\left(V^{G}, F^{G} ; E\right)$, then we have again a short exact sequence:

$$
0 \rightarrow \psi^{-\infty}\left(V^{G}, F^{G} ; E\right) \hookrightarrow \psi^{\infty}\left(V^{G}, F^{G} ; E\right) \longrightarrow \mathcal{A}\left(V^{G}, F^{G} ; E\right) \rightarrow 0 .
$$

The Schwartz theorem enables us to identify the ideal $\psi^{-\infty}\left(V^{G}, F^{G} ; E\right)$ with the smooth convolution algebra $C_{c}^{\infty}\left(\mathcal{G}_{X} ; \operatorname{End}(E)\right)$. Therefore, and using the boundary map in $K$-theory as we did for the flat bundle $(V, F)$, we define the equivariant $G$ index $\operatorname{Ind}_{G}(P)$ of a longitudinally elliptic pseudodifferential operator $P$ on $\left(V^{G}, F^{G}\right)$ to be the image under this boundary map and the Morita equivalence, in the group $K^{G}\left(C_{c}^{\infty}\left(\mathcal{G}_{X}\right)\right)$, of the $K$-theory class $[\sigma(P)] \in K_{1}^{G}\left(\mathcal{A}\left(V^{G}, F^{G} ; E\right)\right)$ of the principal symbol $\sigma(P)$ of $P$. We point out that the principal symbol $\sigma(P)$ of $P$ also defines a homotopy class in $K_{G}^{0}\left(F^{G, *}\right)$, where $F^{G \text {,* }}$ is the colongitudinal bundle to the foliated manifold $\left(V^{G}, F^{G}\right)$. The equivariant $K$-theory of the algebra $C_{c}^{\infty}\left(\mathcal{G}_{X}\right)$ is isomorphic to the equivariant $K$-theory of the crossed product algebra $C^{\infty}\left(A^{G}\right) \rtimes \Gamma$. Therefore, using the clopen inclusion of $A^{G}$ in $\mathcal{T}$ together with the Morita equivalences, we can view the $G$-index of any longitudinally elliptic pseudodifferential $G$-operator $P$ on $\left(V^{G}, F^{G}\right)$ as an element of the group $K^{G}\left(C_{c}^{\infty}(\mathcal{G})\right)$ or $K^{G}\left(C^{*}(V, F)\right)$.

We are now in position to state the Lefschetz theorem: 
Theorem 2. 6] The Lefschetz class $\mathbb{L}(g ; P)$ belongs to the image of $K^{G}\left(C_{c}^{\infty}\left(\mathcal{G}_{X}\right)\right)_{g}$ in $K^{G}\left(C_{c}^{\infty}(\mathcal{G})\right)_{g}$. More precisely, we have the following Lefschetz formula:

$$
\mathbb{L}(g ; P)=\left(\operatorname{Ind} \otimes R(G)_{g}\right)\left(\frac{i^{*}[\sigma(P)]}{\lambda_{-1}\left(N^{G} \otimes \mathbb{C}\right)}\right) \quad \in K^{G}\left(C_{c}^{\infty}(\mathcal{G})\right)_{g},
$$

where $i^{*}: K_{G}\left(F^{*}\right)_{g} \rightarrow K_{G}\left(F^{G, *}\right)_{g}$ is the restriction morphism.

In the RHS of the formula in Theorem 2, the fraction means that the class $\lambda_{-1}\left(N^{G} \otimes \mathbb{C}\right)$ becomes a unit when viewed in $K_{G}\left(V^{G}\right)_{g}$, and the expression Ind $\otimes R(G)_{g}$ means that we have used the identifications

$$
K_{G}\left(F^{G}\right)_{g} \simeq K\left(F^{G}\right) \otimes R(G)_{g} \text { and } K^{G}\left(C_{c}^{\infty}\left(\mathcal{G}_{X}\right)\right)_{g} \simeq K\left(C_{c}^{\infty}\left(\mathcal{G}_{X}\right)\right) \otimes R(G)_{g},
$$

to replace $\operatorname{Ind}_{G}$ on $\left(V^{G}, F^{G}\right)$ by $\operatorname{Ind} \otimes R(G)_{g}$.

The first nontrivial application of such a theorem concerns the existence of fixed points under the action of $G$. Since the $K$-theory groups which are involved are not easy to compute in general, we need to construct additive maps from the $G$ equivariant $K$-theory of the algebra $C_{c}^{\infty}(\mathcal{G})$ to the scalars, which in addition must agree with the localization at the prime ideal associated with $g$, used in Theorem 2

\section{INVARIANT CURRENTS AND CYCLIC COCYCLES}

This section is devoted to the construction of a morphism from the de Rham homology of invariant currents on the smooth compact manifold $\mathcal{T}$ to the cyclic cohomology of the smooth convolution algebra of the Lie groupoid $\mathcal{G}$ described in the previous section. This construction admits an independent interest and should give applications in relation, for instance, with the results of Hurder, Kaminker and Douglas on foliated bundles 22 .

Let again $(V, F)$ be the foliated bundle defined in the previous section. We denote by $\Omega^{k}(\mathcal{T})$ the space of differential $k$-forms on the manifold $\mathcal{T}$. So $\Omega^{k}(\mathcal{T})$ is endowed with a smooth action of the discrete group $\Gamma$ corresponding to the pullback of differential forms. A transverse compactly supported differential $k$-form on the groupoid $\mathcal{G}$ is a smooth $\Gamma$-invariant section of the bundle $\Lambda\left(\nu^{*}\right)$ of exterior powers of the transverse bundle $\nu$, with $\Gamma$-compact support. This means that when viewed as a smooth section over $\mathcal{G}$, the differential form is compactly supported. If we denote by $\pi_{2}$ the projection of $\tilde{M} \times \tilde{M} \times \mathcal{T}$ onto $\mathcal{T}$, then a transverse compactly supported differential $k$-form on $\mathcal{G}$ corresponds bijectively to a smooth section over the groupoid $\tilde{M} \times \tilde{M} \times \mathcal{T}$ of the vector bundle $\pi_{2}^{*} \Lambda^{k}(\mathcal{T})$ that is $\Gamma$-invariant with $\Gamma$-compact support. In the same way, for any $F$-transverse distribution $\mathcal{H}$ over $V$, we can view a transverse differential form as a smooth $\Gamma$-invariant section over $\mathcal{G}$ of the bundle $r^{*} \Lambda\left(\mathcal{H}^{*}\right)$, which has $\Gamma$-compact support.

While the "best" realization of the transverse bundle seems to be given by the vertical tangent bundle to the fibration $V \rightarrow M$, this transverse distribution will not be equivariant for longitudinal Lie group actions in general. Therefore, we need to work with a transverse distribution $\mathcal{H}$, which is not necessarily integrable for instance. Recall that the holonomy pseudogroup of the foliated manifold $(V, F)$ acts by local diffeomorphisms between transversals to $F$. A holonomy-invariant current corresponds to a family of currents $\left(C_{T}\right)_{T}$ where $T$ runs through the transversals of $(V, F)$, which is invariant under the action of the holonomy pseudogroup. For more details about holonomy-invariant currents, we refer the reader to [23]. 
Lemma 1. Any closed $\Gamma$-invariant $k$-current $C$ on the manifold $\mathcal{T}$ yields a holonomy-invariant current on the foliated manifold $(V, F)$.

Proof. We fix a fibre $\mathcal{T}$ of the fibration $V \rightarrow M$; so $\mathcal{T}$ is transverse to the leaves of $(V, F)$ and cuts all the leaves. If $T$ is any open transverse submanifold to the foliation $(V, F)$ that is contained in a distinguished chart, then there exists a path $\gamma$ drawn in a leaf such that $s(\gamma) \in T, r(\gamma) \in \mathcal{T}$ and the local holonomy diffeomorphism induced by $\gamma$ sends $T$ onto some open submanifold $\mathcal{T}_{\gamma}$ of $\mathcal{T}$. Now, given a smooth compactly supported differential form $\omega$ on $T$, we can use the holonomy transformation induced by $\gamma$ to pull back $\omega$ to a differential form $\omega^{\prime}$ on $\mathcal{T}$ and we set

$$
\left\langle C_{T}, \omega\right\rangle:=\left\langle C, \omega^{\prime}\right\rangle .
$$

If $\gamma^{\prime}$ is another path drawn in a leaf and whose holonomy diffeomorphism sends $T$ onto some open submanifold $\mathcal{T}_{\gamma^{\prime}}$ of $\mathcal{T}$ and the differential form $\omega$ onto $\omega^{\prime \prime}$, then we can easily assume that the source of $\gamma$ coincides with the source of $\gamma^{\prime}$ and construct a path in a leaf whose source and range belong to $\mathcal{T}$, and whose holonomy local diffeomorphism sends $\mathcal{T}_{\gamma}$ onto $\mathcal{T}_{\gamma^{\prime}}$ and $\omega^{\prime}$ onto $\omega^{\prime \prime}$. But this last local diffeomorphism is given by some element of the group $\Gamma$; therefore, the $\Gamma$-invariance of $C$ shows that $C_{T}$ is a well-defined current on $T$. It is a consequence of the definition of the currents $\left(C_{T}\right)_{T}$ that they yield a holonomy-invariant transverse current on the foliated manifold $(V, F)$.

A classical construction enables us now to associate to any holonomy-invariant transverse current on $(V, F)$ and any transverse distribution $\mathcal{H}$, a cyclic cocycle on the smooth convolution algebra $C_{c}^{\infty}(\mathcal{G})$. More precisely, we set [16]:

$$
\Omega^{k}:=C_{c}^{\infty}\left(\mathcal{G}, r^{*} \Lambda^{k}\left(\mathcal{H}^{*}\right)\right) \text {. }
$$

The graded space $\Omega^{*}=\bigoplus_{k \geq 0} \Omega^{k}$ is then endowed with the structure of a graded algebra defined by

$$
\left(\omega \omega^{\prime}\right)\left[\tilde{m}, \tilde{m}^{\prime}, t\right]:=\int_{\tilde{M}} \omega[\tilde{m}, \tilde{n}, t] \wedge[\tilde{m}, \tilde{n}, t] \omega^{\prime}\left[\tilde{n}, \tilde{m}^{\prime}, t\right] d \tilde{n} .
$$

We have used in this definition the action of the groupoid $\mathcal{G}$ on the vector bundle $\bigoplus_{k \geq 0} r^{*} \Lambda^{k}\left(\mathcal{H}^{*}\right)$ given by holonomy. So $\omega^{\prime}\left[\tilde{n}, \tilde{m}^{\prime}, t\right]$ belongs to $\Lambda^{k}\left(\mathcal{H}_{[\tilde{n}, t]}^{*}\right)$ and the path $[\tilde{m}, \tilde{n}, t]$ acts by holonomy from $\Lambda^{k}\left(\mathcal{H}_{[\tilde{n}, t]}^{*}\right)$ to $\Lambda^{k}\left(\mathcal{H}_{[\tilde{m}, t]}^{*}\right)$.

Let us recall now the integration along the leaves, as defined by Haefliger in [23]. Let $U$ be a fundamental open domain for the action of $\Gamma$ by deck transformations on $\tilde{M}$. There exists a smooth foliated embedding of the manifold $U \times \mathcal{T}$ with its trivial foliation by copies of $U$, inside $V$ with the foliation $F$ such that $V \backslash(U \times \mathcal{T})$ is longitudinally Borel negligible. By using a finite open cover $\left(\mathcal{T}_{i}\right)_{i}$ of $\mathcal{T}$, we obtain a finite open cover $\left(U \times \mathcal{T}_{i}\right)_{i}$ of $U \times \mathcal{T}$ by open distinguished charts. Moreover, inside each open set $W_{i}:=U \times \mathcal{T}_{i}$, we can assume that there exists a smooth transversal $T_{i}$ that is tangent to the distribution $\mathcal{H}$ inside $W_{i}$ and that cuts all the plaques of $W_{i}$. Hence we have

$$
W_{i} \simeq U \times \mathcal{T}_{i} \simeq U \times T_{i}, \quad \forall i \in I .
$$

We can even assume that the transversal $T:=\bigcup_{i} T_{i}$ is a smooth (disconnected in general) transverse submanifold of $V$. Then $T$ cuts all the leaves of $(V, F)$. Now if $\omega$ is a smooth differential form on the groupoid associated with the trivial foliation of $W_{i}$, then we can integrate the restriction of $\omega$ to the unit manifold, on the plaques 
of $W_{i}$ and obtain a smooth differential form $\alpha$ on $T_{i}$. Evaluation of the holonomyinvariant current $C_{T}$ associated with $C$ and constructed above, on the differential form $\alpha$ yields a pairing of $C$ with $\omega$. A partition of unity argument then enables us to construct the Haefliger map [23]:

$$
\phi_{U}^{\mathcal{H}}: \Omega^{k} \longrightarrow \Omega^{k}(T) .
$$

If we denote by $\phi^{\mathcal{H}}$ the composite of $\phi_{U}^{\mathcal{H}}$ with the projection $\Omega^{k}(T) \rightarrow \Omega^{k}(\mathcal{H} / F)$, then $\phi^{\mathcal{H}}$ is independent of the choices fixed in its definition, see [23]. Here we have denoted by $\Omega^{k}(\mathcal{H} / F)$ the quotient space of $\Omega^{k}(T)$ by the (nonclosed in general) subspace generated by elements $h^{*} \omega-\omega$ where $h$ is a local holonomy diffeomorphism on $T$ and $\omega$ is a smooth compactly supported differential form on $T$. The notation $\Omega^{k}(\mathcal{H} / F)$ is made legitimate by the fact that if we change $T$ into some other complete transversal $T^{\prime}$ that is tangent to $\mathcal{H}$, then the quotient spaces are naturally isomorphic; see again [23].

By using a connection on the vector bundle $\mathcal{H}$, one constructs locally a differential on the sections of the exterior powers $\Lambda\left(\mathcal{H}^{*}\right)$. By using a partition of unity, we get in this way a global differential $d_{\mathcal{H}}$ on $V$. The same construction over the groupoid $\mathcal{G}$ yields a differential that we shall also denote by $d_{\mathcal{H}}$. Note that $\mathcal{G}$ is also endowed with a foliation $F_{\mathcal{G}}$ whose leaves are the projections in $\mathcal{G}$ of the submanifolds $\tilde{M} \times \tilde{M} \times\{t\}$ of $\tilde{M} \times \tilde{M} \times T$ when $t$ runs through $T$. The de Rham differential on the manifold $\mathcal{G}$ thus splits with respect to the transverse distribution $\mathcal{H}$ into [34]:

$$
d=d_{F}+d_{\mathcal{H}}+\partial,
$$

where $d_{F}$ is the longitudinal de Rham differential and $\partial$ is an extra differential which has bidegree $(-1,2)$ with respect to the splitting $F_{\mathcal{G}} \oplus \mathcal{H}=T \mathcal{G}$; see also 16, page 266]. The transverse differential $d_{\mathcal{H}}$ sends $\Omega^{k}$ to $\Omega^{k+1}$ and is a graded derivation of the graded algebra $\Omega^{*}$. Moreover, by using the curvature of the connection used to define $d_{\mathcal{H}}$, it is easy to show that the square $d_{\mathcal{H}}^{2}$ of $d_{\mathcal{H}}$ is given by the commutator with a section $\Theta$ of $\Lambda^{2} \mathcal{H}^{*}$ :

$$
d_{\mathcal{H}}^{2}(\omega)=\Theta \omega-\omega \Theta, \quad \forall \omega \in \Omega^{*},
$$

such that $d_{\mathcal{H}}(\Theta)=0$; see again [16, page 767]. To build out of the graded algebra $\left(\Omega^{*}, d_{\mathcal{H}}\right)$ a new graded differential algebra $(\tilde{\Omega}, \tilde{d})$ such that $\tilde{d}^{2}=0$, we shall use Connes' trick. More precisely, we adjoin to the algebra $\Omega^{*}$, a formal square-root of $\Theta$ and modify the differential $d_{\mathcal{H}}$ according to the new algebraic structure. This gives a vector space $\tilde{\Omega}:=M_{2}(\Omega)$ with a product given by

$$
\tilde{\omega} * \tilde{\omega}^{\prime}:=\tilde{\omega} \tilde{\Theta} \tilde{\omega}^{\prime},
$$

where

$$
\tilde{\Theta}=\left(\begin{array}{cc}
1 & 0 \\
0 & \Theta
\end{array}\right) .
$$

The new grading on $\tilde{\Omega}$ is then given by

$$
\partial \tilde{\omega}:=\partial \tilde{\omega}_{11}=\partial \tilde{\omega}_{12}+1=\partial \tilde{\omega}_{21}+1=\partial \tilde{\omega}_{22}+2,
$$

and the new differential $\tilde{d}$ is defined by

$$
\tilde{d} \tilde{\omega}:=\left(\begin{array}{cc}
d_{\mathcal{H}} \tilde{\omega}_{11} & d_{\mathcal{H}} \tilde{\omega}_{12} \\
-d_{\mathcal{H}} \tilde{\omega}_{21} & -d_{\mathcal{H}} \tilde{\omega}_{22}
\end{array}\right)+\left(\begin{array}{cc}
0 & -\Theta \\
1 & 0
\end{array}\right) \tilde{\omega}+(-1)^{\partial \tilde{\omega}} \tilde{\omega}\left(\begin{array}{cc}
0 & 1 \\
-\Theta & 0
\end{array}\right) .
$$


One then shows, by straightforward computation for instance, that $\tilde{d}^{2}=0$ [16, page 229]. For simplicity, we shall also and from now on denote by $\tilde{\omega} \tilde{\omega}^{\prime}$ the product $\tilde{\omega} * \tilde{\omega}^{\prime}$ and by $d$ the differential $\tilde{d}$. The imbedding of $\Omega^{*}$ in $\tilde{\Omega}^{*}$ is defined by

$$
\omega \hookrightarrow\left(\begin{array}{cc}
\omega & 0 \\
0 & 0
\end{array}\right) .
$$

It will be used in the sequel to view the elements of $\Omega^{*}$ as elements of $\tilde{\Omega}^{*}$. We define $\operatorname{Tr}_{\Theta}: \tilde{\Omega}^{k} \rightarrow \Omega^{k}$ by

$$
\operatorname{Tr}_{\Theta}(\tilde{\omega}):=\tilde{\omega}_{11}-(-1)^{k} \tilde{\omega}_{22} \Theta .
$$

Now we can extend $\phi^{\mathcal{H}}$ to $\tilde{\Omega}$ by setting

$$
\psi_{\Theta}^{\mathcal{H}}:=\phi^{\mathcal{H}} \circ \operatorname{Tr}_{\Theta}: \tilde{\Omega}^{k} \rightarrow \Omega^{k}(\mathcal{H} / F) .
$$

Proposition 1. With the above notation we have:

(i) The functional $\psi_{\Theta}^{\mathcal{H}}$ is a graded trace on the graded algebra $\tilde{\Omega}$.

(ii) If we also denote by d the differential induced on $\Omega^{*}(\mathcal{H} / F)$ by the de Rham differential, then we have

$$
\psi_{\Theta}^{\mathcal{H}} \circ d=d \circ \psi_{\Theta}^{\mathcal{H}} .
$$

Proof. (i) For $\omega \in \Omega^{j}$ and for $\omega^{\prime} \in \Omega^{j^{\prime}}$, we need to show that

$$
\phi^{\mathcal{H}}\left(\omega \omega^{\prime}\right)=(-1)^{j j^{\prime}} \phi^{\mathcal{H}}\left(\omega^{\prime} \omega\right) .
$$

If this equality is proved, then we simply observe that for any $\left(\tilde{\omega}, \tilde{\omega}^{\prime}\right) \in \tilde{\Omega}^{k} \times \tilde{\Omega}^{k^{\prime}}$, we have

$$
\begin{gathered}
\operatorname{Tr}_{\Theta}\left[\tilde{\omega}, \tilde{\omega}^{\prime}\right]=\left[\tilde{\omega}_{11}, \tilde{\omega}_{11}^{\prime}\right]+\left[\tilde{\omega}_{12} \Theta, \tilde{\omega}_{21}^{\prime}\right]+(-1)^{k k^{\prime}+1}\left[\tilde{\omega}_{12}^{\prime} \Theta, \tilde{\omega}_{21}\right] \\
+(-1)^{k+k^{\prime}+1}\left[\tilde{\omega}_{22} \Theta, \tilde{\omega}_{22}^{\prime} \Theta\right]
\end{gathered}
$$

where the commutators are graded ones.

Now let us prove Relation (44). Fix then $\omega \in \Omega^{j}$ and $\omega^{\prime} \in \Omega^{j^{\prime}}$. We can assume that $\omega$ is supported inside a trivial chart of $\mathcal{G}$. More precisely, we can assume that $\omega$ is supported in an open subset of $\mathcal{G}$ of the type $W_{i} \times_{\gamma} W_{j}$, where $W_{i} \simeq U \times T_{i}$ and $W_{j} \simeq U \times T_{j}$ are two distinguished charts for the foliation $(U \times \mathcal{T}, F)$ and where $W_{i} \times_{\gamma} W_{j}$ denotes the subset of paths near the path $\gamma$ with source in $W_{i}$ and range in $W_{j}$ and drawn inside a tunnel around $\gamma$ [14]. The holonomy local transformation of $\gamma$ is defined on $W_{i}$ and identifies any transversal inside $W_{i}$ with a transversal inside $W_{j}$. The chart $W_{i} \times_{\gamma} W_{j}$ of $\mathcal{G}$ is then diffeomorphic to $U \times U \times T_{i}$ by using the holonomy local transformation associated with $\gamma$. Therefore, we have $\left(\omega \omega^{\prime}\right)(x)=0$ if $x \notin W_{j}$ and for $x \in W_{j}$ and using the trivializations, we can write with an abuse of notation:

$$
\left(\omega \omega^{\prime}\right)\left(x=\left(w^{\prime}, t\right)\right)=\int_{U} \omega\left(w^{\prime}, w, t\right) \wedge\left(w^{\prime}, w, t\right)\left(\omega^{\prime}\left(w, w^{\prime}, t\right)\right) d w .
$$

In the same way, we get $\left(\omega^{\prime} \omega\right)(x)=0$ if $x \notin W_{i}$ and for $x \in W_{i}$, we have

$$
\left(\omega^{\prime} \omega\right)(x=(w, t))=\int_{U} \omega^{\prime}\left(w, w^{\prime}, t\right) \wedge\left(w, w^{\prime}, t\right)\left(\omega\left(w^{\prime}, w, t\right)\right) d w^{\prime} .
$$

Therefore, we obtain

$$
\left[\phi^{\mathcal{H}}\left(\omega \omega^{\prime}\right)\right](t)=\int_{U \times U} \omega\left(w^{\prime}, w, t\right) \wedge\left(w^{\prime}, w, t\right)\left(\omega^{\prime}\left(w, w^{\prime}, t\right)\right) d w d w^{\prime},
$$


while

$$
\left[\phi^{\mathcal{H}}\left(\omega^{\prime} \omega\right)\right](t)=\int_{U \times U} \omega^{\prime}\left(w, w^{\prime}, t\right) \wedge\left(w, w^{\prime}, t\right)\left(\omega\left(w^{\prime}, w, t\right)\right) d w^{\prime} d w .
$$

Hence we see that as compactly supported differential forms on the transversal $T_{i}$ in $W_{i}$ and the transversal $T_{j}$ in $W_{j}$ respectively, we have

$$
\phi_{U}^{\mathcal{H}}\left(\omega \omega^{\prime}\right)=(-1)^{j j^{\prime}} \varphi_{\gamma}^{*} \phi_{U}^{\mathcal{H}}\left(\omega^{\prime} \omega\right),
$$

where $\varphi_{\gamma}$ is the holonomy transformation induced by the fixed path $\gamma$. Thus the proof of (i) is complete.

(ii) The computation of $\operatorname{Tr}_{\Theta} \circ d$ for a $(k-1)$-homogeneous element $\tilde{\omega}$ of $\tilde{\Omega}$ gives

$$
\left(\operatorname{Tr}_{\Theta} \circ d\right)(\tilde{\omega})=d_{\mathcal{H}} \tilde{\omega}_{11}+(-1)^{k} d_{\mathcal{H}} \tilde{\omega}_{22} \Theta-d_{\mathcal{H}}^{2} \tilde{\omega}_{21} .
$$

On the other hand, if $i^{*}$ is the restriction to the unit manifold $\mathcal{G}^{(0)}=V$, then it is easy to deduce from the definitions that with respect to the splittings over $\mathcal{G}$ and over $V$ corresponding to the same distribution $\mathcal{H}$, we have

$$
d_{\mathcal{H}} \circ i^{*}=i^{*} \circ d_{\mathcal{H}} .
$$

But one can also easily check that [23]:

$$
\phi^{\mathcal{H}} \circ d=d \circ \phi^{\mathcal{H}} \quad \text { and } \quad \phi^{\mathcal{H}} \circ d=\phi^{\mathcal{H}} \circ d_{\mathcal{H}} .
$$

Therefore, we can deduce that

$$
\phi^{\mathcal{H}} \circ d_{\mathcal{H}}=d \circ \phi^{\mathcal{H}}
$$

and finally,

$$
\begin{aligned}
\left(\phi^{\mathcal{H}} \circ \operatorname{Tr}_{\Theta} \circ d\right)(\tilde{\omega}) & =\left(d \circ \phi^{\mathcal{H}}\right) \tilde{\omega}_{11}+(-1)^{k}\left(d \circ \phi^{\mathcal{H}}\right) \tilde{\omega}_{22} \Theta \\
& =\left(d \circ \phi^{\mathcal{H}}\right)\left(\tilde{\omega}_{11}-(-1)^{k-1} \tilde{\omega}_{22} \Theta\right) .
\end{aligned}
$$

Theorem 3. Any $\Gamma$-invariant closed $k$-current $C$ on $\mathcal{T}$ and any transverse distribution $\mathcal{H}$ induce a cyclic $k$-cocycle $\tau_{C}^{\mathcal{H}}$ on the convolution algebra $C_{c}^{\infty}(\mathcal{G})$ defined for $\left(f_{0}, \cdots, f_{k}\right) \in C_{c}^{\infty}(\mathcal{G})^{k+1}$ by Connes' formula:

$$
\tau_{C}^{\mathcal{H}}\left(f_{0}, \cdots, f_{k}\right):=\left\langle C, \psi_{\Theta}^{\mathcal{H}}\left(f_{0} d f_{1} \cdots d f_{k}\right)\right\rangle,
$$

where $d$ is the differential constructed above, out of the transverse differential $d_{\mathcal{H}}$.

Proof. We shall use the graded algebra $\tilde{\Omega}$. We thus define for $\left(f_{0}, \cdots, f_{k}\right) \in$ $C_{c}^{\infty}(\mathcal{G})^{k+1}$ :

$$
\tau_{C}^{\mathcal{H}}\left(f_{0}, \cdots, f_{k}\right):=\left\langle C, \psi_{\Theta}^{\mathcal{H}}\left(f_{0} d f_{1} \cdots d f_{k}\right)\right\rangle .
$$

Because the current $C$ is closed and using Proposition 1, we deduce that the map

$$
\tilde{\omega} \longmapsto\left\langle C,\left(\phi \circ \operatorname{Tr}_{\Theta}\right)(\tilde{\omega})\right\rangle
$$

is a graded trace on $\tilde{\Omega}$. This trace is, in addition, closed; so we deduce by classical arguments that $\tau_{C}^{\mathcal{H}}$ is a cyclic cocycle on the algebra $C_{c}^{\infty}(\mathcal{G})$, see [17]. 


\section{LONGITUDINAL EQUIVARIANCE OF INVARIANT CURRENTS}

In this section, we shall prove that any even cyclic cocycle on the algebra $C_{c}^{\infty}(\mathcal{G})$ that arises from some $\Gamma$-invariant closed current on $\mathcal{T}$ and a $G$-equivariant transverse distribution, admits a well-defined pairing with $G$-equivariant $K$-theory and localized $G$-equivariant $K$-theory. Because the pairing obtained in the present section is a general phenomenon, we shall remove the condition used in Section 1 that $G$ is topologically cyclic. Therefore, and in this section only, we shall suppose that $G$ is a compact Lie group that acts by $F$-preserving isometries on $V$ and that $G_{1}$ is any subgroup that acts by leaf-preserving isometries. Our case of interest corresponds as in Section 1, to $G_{1}$ being the dense subgroup of powers of $g$, where $g$ is now assumed to preserve the leaves.

Hence, we are given a lift to $\tilde{M} \times \mathcal{T}$ of the action of $G_{1}$, which is defined by a group homomorphism $\beta$ from $G_{1}$ to the group $\operatorname{Aut}(\Gamma)$ of automorphisms of $\Gamma$. In Section 1, this homomorphism was completely determined by its value at $g$, and we denoted by $\beta$ the value at $\tilde{g}$. So, any $g \in G_{1}$ is induced by a smooth diffeomorphism $\tilde{g}$ of $\tilde{M} \times \mathcal{T}$ and is a leaf-preserving diffeomorphism. Therefore, $\tilde{g}$ is defined by

$$
\tilde{g}(\tilde{m}, t):=(g(t)(\tilde{m}), \alpha(g)(t)), \quad \forall(\tilde{m}, t) \in \tilde{M} \times \mathcal{T},
$$

where $g: \mathcal{T} \rightarrow \operatorname{Diff}(\tilde{M})$ is a smooth map from $\mathcal{T}$ to the diffeomorphism group of the manifold $\tilde{M}$ and $\alpha$ is a smooth action of $G_{1}$ on $\mathcal{T}$.

Now since the group $G_{1}$ preserves the leaves, the action $\alpha$ is actually given by $\varrho \circ \delta$ where $\delta$ is some homomorphism from $G_{1}$ to $\Gamma$. Therefore, and using the $\beta$ equivariance of $\tilde{g}$, see Equation (2), we deduce that we can assume that $\beta$ takes values in inner automorphisms of $\Gamma$. More precisely, we can assume that

$$
\beta(g, \gamma)=\delta(g) \gamma \delta(g)^{-1}, \quad \forall(g, \gamma) \in G_{1} \times \Gamma .
$$

This discussion shows that if we replace the map $g: \mathcal{T} \rightarrow \operatorname{Diff}(\tilde{M})$ which defines the action of $\tilde{g}$, by the map

$$
g^{\prime}: \mathcal{T} \longrightarrow \operatorname{Diff}(\tilde{M}) \text { given by } g^{\prime}(t)(\tilde{m}):=g(t)(\tilde{m}) \delta(g),
$$

then we get a $\Gamma$-equivariant lift of $g$.

To sum up, in the case where $g$ preserves the leaves and replacing $g(t)$ by $g^{\prime}(t)$ if necessary, we can choose a $\Gamma$-equivariant lift of $g$ given by

$$
\tilde{g}(\tilde{m}, t):=(g(t)(\tilde{m}), t), \quad \forall(\tilde{m}, t) \in \tilde{M} \times \mathcal{T},
$$

where $g\left(\varrho\left(\gamma^{-1}\right)\right)(\tilde{m} \gamma)=g(t)(\tilde{m}) \gamma$ for any $\gamma \in \Gamma$. This means that we can take $\beta(g, \gamma)=\gamma$ for any $(g, \gamma) \in G_{1} \times \Gamma$. Hence the action can be reduced to an action in the leaf direction by means of the smooth map $g: \mathcal{T} \rightarrow \operatorname{Diff}(\tilde{M})$ which is $\Gamma$ equivariant and $\alpha$ can be taken to be equal to the trivial action. Under these assumptions, we can now define smooth diffeomorphisms of the manifold $\mathcal{G}$ by setting

$$
\begin{array}{r}
\left.L(g)\left[\tilde{m}, \tilde{m}^{\prime}, t\right]:=\left[g(t)^{-1}(\tilde{m}), \tilde{m}^{\prime}, t\right], R(g)\left[\tilde{m}, \tilde{m}^{\prime}, t\right]:=[\tilde{m}), g(t)\left(\tilde{m}^{\prime}\right), t\right] \\
\text { and } f_{g}\left[\tilde{m}, \tilde{m}^{\prime}, t\right]:=\left[g(t)(\tilde{m}), g(t)\left(\tilde{m}^{\prime}\right), t\right] .
\end{array}
$$

These diffeomorphisms induce automorphisms of the algebra $C_{c}^{\infty}(\mathcal{G}) \simeq \Omega^{0}$. We fix again a $\Gamma$-invariant Lebesgue measure $d \tilde{m}$ on $\tilde{M}$ that is, in addition, $G_{1}$-invariant. We then define all the integrals with respect to this measure. We denote by ${ }^{g} k$, 
by $k^{g}$ and by $g k$ the respective induced actions of $g$ on $k \in \Omega^{0}$. So we set, more precisely,

$$
\begin{aligned}
{ }^{g} k\left[\tilde{m}, \tilde{m}^{\prime}, t\right]:=k\left[\tilde{m}, g(t)\left(\tilde{m}^{\prime}\right), t\right], \quad k^{g}\left[\tilde{m}, \tilde{m}^{\prime}, t\right]:=k\left[g(t)^{-1}(\tilde{m}), \tilde{m}^{\prime}, t\right] \\
\text { and } g k\left[\tilde{m}, \tilde{m}^{\prime}, t\right]:=k\left[g(t)^{-1}(\tilde{m}), g(t)^{-1}\left(\tilde{m}^{\prime}\right), t\right] .
\end{aligned}
$$

The measure $d \tilde{m}$ on $\tilde{M}$ induces a Haar system on the groupoid $\mathcal{G}$. Since $d \tilde{m}$ is invariant under the action of the Lie group $G_{1}$, the transformation $\psi(g)$ for $g \in G_{1}$, defined on $L^{2}\left(\mathcal{G}^{[\tilde{m}, t]} \simeq \tilde{M}, d \tilde{m}\right)$ by

$$
\psi(g)(\xi)\left[\tilde{m}, \tilde{m}^{\prime}, t\right]:=\xi\left[\tilde{m}, g(t)\left(\tilde{m}^{\prime}\right), t\right]
$$

is unitary and satisfies

$$
\psi(g) \circ \pi(k)=\pi\left({ }^{g} k\right) \quad \text { and } \quad \pi(k) \circ \psi(g)=\pi\left(k^{g}\right) .
$$

Therefore, the action of $G_{1}$ on $C_{c}^{\infty}(\mathcal{G})$ is also implemented by the unitaries $\psi(g)$, i.e.,

$$
\psi(g) \circ \pi(k) \circ \psi\left(g^{-1}\right)=\pi(g k) .
$$

In the above relations, $\pi$ denotes, for any $x \in V$, the regular representation of $C_{c}^{\infty}(\mathcal{G})$ corresponding to $x$ in the $L^{2}$ space of the manifold $\left(\mathcal{G}^{x} \simeq \tilde{M}, d \tilde{m}\right)$.

We fix as in Section 1, a $G$-invariant metric on $V$, obtained for instance by averaging a given one with respect to the normalized Haar measure of $G$. The orthogonal distribution to the longitudinal vector bundle $F$ in $T V$ with respect to this metric is then a transverse distribution that we denote by $\mathcal{H}$. We then denote by $\Omega^{*}$ the graded differential algebra associated with $\mathcal{H}$ and by $\tilde{\Omega}^{*}$ the associated one constructed in Section 2. Note that for any $\omega \in \Omega^{*}$, a new differential form ${ }^{g_{\omega}} \in \Omega^{*}$ is defined by setting

$$
\left({ }^{g} \omega\right)\left[\tilde{m}, \tilde{m}^{\prime}, t\right]:=\omega\left[\tilde{m}, g(t)\left(\tilde{m}^{\prime}\right), t\right] .
$$

Remark 2. Instead of fixing the measure $d \tilde{m}$ on $\tilde{M}$, we could use half-densities. Then we simply use the action on the vector bundle $|\Lambda|^{1 / 2}(F)$ of longitudinal halfdensities over $V$, see also [7].

Lemma 2. (1) For any $f \in \Omega^{0}$, any $\omega \in \Omega^{k}$, any $g \in G_{1}$ and any $\left[\tilde{m}, \tilde{m}^{\prime}, t\right] \in \mathcal{G}$, we have

$$
\left(\left(f^{g}\right) \omega\right)\left[\tilde{m}, \tilde{m}^{\prime}, t\right]=\left[\tilde{m}, g(t)^{-1}(\tilde{m}), t\right]\left(\left(f\left({ }^{g} \omega\right)\right)\left[g(t)^{-1}(\tilde{m}), g(t)^{-1}\left(\tilde{m}^{\prime}\right), t\right]\right) .
$$

Therefore, $\phi^{\mathcal{H}}\left(\left(f^{g}\right) \omega\right)=\phi^{\mathcal{H}}\left(f\left({ }^{g} \omega\right)\right)$ in $\Omega^{k}(\mathcal{H} / F)$.

(2) For any $\left(\omega_{1}, \omega_{2}\right) \in \Omega^{*} \times \Omega^{*}$, we have

$$
{ }^{g}\left(\omega_{1} \omega_{2}\right)=\omega_{1}\left({ }^{g} \omega_{2}\right)
$$


Proof. (1) Let $\left(\tilde{m}, \tilde{m}^{\prime}, t\right) \in \tilde{M} \times \tilde{M} \times \mathcal{T}$ be fixed. We have

$$
\begin{aligned}
\left(\left(f^{g}\right) \omega\right)\left[\tilde{m}, \tilde{m}^{\prime}, t\right]= & \int_{\tilde{M}} f^{g}[\tilde{m}, \tilde{n}, t][\tilde{m}, \tilde{n}, t]\left(\omega\left[\tilde{n}, \tilde{m}^{\prime}, t\right]\right) d \tilde{n} \\
= & \int_{\tilde{M}} f\left[g(t)^{-1}(\tilde{m}), \tilde{n}, t\right][\tilde{m}, \tilde{n}, t]\left(\omega\left[\tilde{n}, \tilde{m}^{\prime}, t\right]\right) d \tilde{n} \\
= & \int_{\tilde{M}} f\left[g(t)^{-1}(\tilde{m}), \tilde{n}, t\right]\left[\tilde{m}, g(t)^{-1}(\tilde{m}), t\right] \\
& {\left[g(t)^{-1}(\tilde{m}), \tilde{n}, t\right]\left(\omega\left[\tilde{n}, \tilde{m}^{\prime}, t\right]\right) d \tilde{n} } \\
= & {\left[\tilde{m}, g(t)^{-1}(\tilde{m}), t\right] \int_{\tilde{M}} f\left[g(t)^{-1}(\tilde{m}), \tilde{n}, t\right] } \\
& {\left[g(t)^{-1}(\tilde{m}), \tilde{n}, t\right]\left(\omega\left[\tilde{n}, \tilde{m}^{\prime}, t\right]\right) d \tilde{n} } \\
= & {\left[\tilde{m}, g(t)^{-1}(\tilde{m}), t\right] \int_{\tilde{M}} f\left[g(t)^{-1}(\tilde{m}), \tilde{n}, t\right] } \\
& {\left[g(t)^{-1}(\tilde{m}), \tilde{n}, t\right]\left({ }^{g} \omega\left[\tilde{n}, g(t)^{-1}\left(\tilde{m} \tilde{m}^{\prime}\right), t\right]\right) d \tilde{n} } \\
= & {\left[\tilde{m}, g(t)^{-1}(\tilde{m}), t\right]\left(\left(f\left({ }^{g} \omega\right)\right)\left[g(t)^{-1}(\tilde{m}), g(t)^{-1}\left(\tilde{m}^{\prime}\right), t\right]\right) . }
\end{aligned}
$$

We deduce that, in restriction to any transversal $\left\{\left[g\left(t^{\prime}\right)^{-1}(\tilde{m}), t^{\prime}\right], t^{\prime}\right.$ close to $\left.t\right\}$ around $\left[g(t)^{-1}(\tilde{m}), t\right]$, the differential form $\left(f^{g}\right) \omega$ is equal to the pull-back by $g$ of the differential form $f\left({ }^{g} \omega\right)$. Since the holonomy transformation induced by $\left[\tilde{m}, g(t)^{-1}(\tilde{m}), t\right]$ on this small transversal around $\left[g(t)^{-1}(\tilde{m}), t\right]$ is exactly given by $g$, we deduce from the above computation that the class $\phi^{\mathcal{H}}\left(\left(f^{g}\right) \omega\right)$ in $\Omega^{k}(\mathcal{H} / F)$ is equal to the class $\phi^{\mathcal{H}}\left(f\left({ }^{g} \omega\right)\right)$.

(2) For any $\left(\tilde{m}, \tilde{m}^{\prime}, t\right) \in \tilde{M} \times \tilde{M} \times \mathcal{T}$, we have

$$
\begin{aligned}
{ }^{g}\left(\omega_{1} \omega_{2}\right)\left[\tilde{m}, \tilde{m}^{\prime}, t\right] & =\left(\omega_{1} \omega_{2}\right)\left[\tilde{m}, g(t)\left(\tilde{m}^{\prime}\right), t\right] \\
& =\int_{\tilde{M}} \omega_{1}[\tilde{m}, \tilde{n}, t] \wedge[\tilde{m}, \tilde{n}, t]\left(\omega_{2}\left[\tilde{n}, g(t)\left(\tilde{m}^{\prime}\right), t\right]\right) d \tilde{n} \\
& =\int_{\tilde{M}} \omega_{1}[\tilde{m}, \tilde{n}, t] \wedge[\tilde{m}, \tilde{n}, t]\left(\left({ }^{g} \omega_{2}\right)\left[\tilde{n}, \tilde{m}^{\prime}, t\right]\right) d \tilde{n} \\
& =\left(\omega_{1}\left({ }^{g} \omega_{2}\right)\right)\left[\tilde{m}, \tilde{m}^{\prime}, t\right] .
\end{aligned}
$$

Theorem 4. Let $C$ be a $\Gamma$-invariant closed $r$-current on the smooth compact manifold $\mathcal{T}$ and let $\tau_{C}^{\mathcal{H}}$ be the associated cyclic cocycle on the smooth convolution algebra $C_{c}^{\infty}(\mathcal{G})$ given by Theorem [3. Then we have, for any $\left(k_{0}, \cdots, k_{r}\right) \in\left(C_{c}^{\infty}(\mathcal{G})\right)^{r+1}$ and any $g \in G_{1}$,

$$
\tau_{C}^{\mathcal{H}}\left(k_{0}^{g}, k_{1}, \cdots, k_{r}\right)=\tau_{C}^{\mathcal{H}}\left(k_{0}, k_{1}, \cdots, k_{r-1},{ }^{g} k_{r}\right) .
$$

Proof. Recall that $\mathcal{H}$ is the orthogonal bundle to $F$ in $T V$ with respect to a $G$ invariant metric on $V$. Therefore, the vector bundle $\mathcal{H}$ is invariant under the action of $G_{1}$. As a simple consequence, we deduce that for any $f \in C_{c}^{\infty}(\mathcal{G})$, we have

$$
d_{\mathcal{H}}\left({ }^{g} f\right)={ }^{g}\left(d_{\mathcal{H}} f\right) .
$$

Note that if we choose a small transversal around $[\tilde{m}, t]$ in $V$ that is tangent to $\mathcal{H}$ and use it to define a local chart, then the action of $g$ becomes $(u, t) \mapsto\left(\varphi_{g}(u), t\right)$ with $\varphi_{g}$ independent of the transverse variable $t$. 
Denote by $\tilde{\omega}$ the element $d k_{1} \cdots d k_{r-1}$ of $\tilde{\Omega}$. The component $\left[\left(k_{0}^{g}\right) \tilde{\omega} d k_{r}\right]_{22}$ of the matrix $\left(k_{0}^{g}\right) \tilde{\omega} d k_{r}$ is obviously trivial. Let us then compute the component $\left[\left(k_{0}^{g}\right) \tilde{\omega} d k_{r}\right]_{11}$ : we get

$$
\left[\left(k_{0}^{g}\right) \tilde{\omega} d k_{r}\right]_{11}=\left(k_{0}^{g}\right) \tilde{\omega}_{11} d_{\mathcal{H}} k_{r}+\left(k_{0}^{g}\right) \tilde{\omega}_{12} \Theta k_{r} .
$$

Hence by using Lemma 2 we obtain

$$
\begin{aligned}
\psi_{\Theta}^{\mathcal{H}}\left(\left(k_{0}^{g}\right) \tilde{\omega} d k_{r}\right) & =\phi^{\mathcal{H}}\left(\left(k_{0}^{g}\right) \tilde{\omega}_{11} d_{\mathcal{H}} k_{r}+\left(k_{0}^{g}\right) \tilde{\omega}_{12} \Theta k_{r}\right) \\
& =\phi^{\mathcal{H}}\left(k_{0}\left[{ }^{g}\left(\tilde{\omega}_{11} d_{\mathcal{H}} k_{r}\right)+{ }^{g}\left(\tilde{\omega}_{12} \Theta k_{r}\right)\right]\right) \\
& =\phi^{\mathcal{H}}\left(k_{0}\left[\tilde{\omega}_{11}\left({ }^{g} d_{\mathcal{H}} k_{r}\right)+\tilde{\omega}_{12} \Theta\left({ }^{g} k_{r}\right)\right]\right) \\
& =\phi^{\mathcal{H}}\left(k_{0}\left[\tilde{\omega}_{11} d_{\mathcal{H}}\left({ }^{g} k_{r}\right)+\tilde{\omega}_{12} \Theta\left({ }^{g} k_{r}\right)\right]\right) .
\end{aligned}
$$

Therefore, we finally get

$$
\psi_{\Theta}^{\mathcal{H}}\left(\left(k_{0}^{g}\right) \tilde{\omega} d k_{r}\right)=\psi_{\Theta}^{\mathcal{H}}\left(k_{0} \tilde{\omega} d\left({ }^{g} k_{r}\right)\right) .
$$

We then apply the holonomy-invariant current induced by $C$ to complete the proof.

Remark 3. The above proof simplifies noticeably when the action of $g$ does not depend on the variable $t \in \mathcal{T}$. In this case, one can take the integrable transverse distribution given by the vertical tangent bundle to the fibration $V \rightarrow M$.

A cochain that satisfies the equivariance relation of Theorem 4 for any $g \in G_{1}$, will be called $G_{1}$-equivariant, compare 28 .

Let now $\kappa: G \rightarrow \operatorname{End}(X)$ be a finite-dimensional unitary representation of the compact Lie group $G$. We define for any elementary tensor $k \otimes T \in C_{c}^{\infty}(\mathcal{G}) \otimes \operatorname{End}(X)$ and any $g \in G_{1}$ :

$$
(k \otimes T)^{g}:=k^{g} \otimes[\kappa(g) \circ T] \quad \text { and } \quad{ }^{g}(k \otimes T):={ }^{g} k \otimes[T \circ \kappa(g)] .
$$

If $\tau$ is a cyclic $k$-cocycle on the convolution algebra $C_{c}^{\infty}(\mathcal{G})$, then we obtain a cyclic $k$-cocycle $\tau \sharp \operatorname{Tr}$ on the algebra $C_{c}^{\infty}(\mathcal{G}) \otimes \operatorname{End}(X)$ by setting [17]:

$$
\begin{aligned}
(\tau \sharp \operatorname{Tr})\left(f_{0} \otimes A_{0}, \cdots, f_{k} \otimes A_{k}\right):=\tau\left(f_{0}, \cdots, f_{k}\right) & \operatorname{Tr}\left(A_{0} \circ \cdots \circ A_{k}\right), \\
& \forall f_{j} \in C_{c}^{\infty}(\mathcal{G}) \text { and } A_{j} \in \operatorname{End}(X) .
\end{aligned}
$$

Moreover, if $\tau$ is $G_{1}$-equivariant, then so is the cocycle $\tau \sharp T r$. Now we can state:

Theorem 5. Let $C$ be a closed $\Gamma$-invariant even current on the manifold $\mathcal{T}$. Assume that $G$ preserves the bundle $F$ and let $X$ be a finite-dimensional representation of the compact Lie group $G$. For any $G$-invariant projection e in $C_{c}^{\infty}(\mathcal{G}) \otimes \operatorname{End}(X)$ and any $g \in G_{1}$, we define

$$
\left\langle\tau_{C}^{g}, e\right\rangle:=\left(\tau_{C}^{\mathcal{H}} \sharp \operatorname{Tr}\right)\left(e^{g}, e, \cdots, e\right) .
$$

Then the following properties hold:

(1) The map $g \mapsto\left\langle\tau_{C}^{g}, e\right\rangle$ is a central function on $G_{1}$.

(2) The map e $\mapsto\left\langle\tau_{C}^{g}, e\right\rangle$ induces an additive map from the $G$-equivariant $K$ theory of the algebra $C_{c}^{\infty}(\mathcal{G})$ to the scalars.

(3) For any fixed conjugacy class $[g]$ in $G_{1},\left\langle\tau_{C}^{[g]}, e\right\rangle$ induces a pairing between the de Rham homology of $\Gamma$-invariant currents on $\mathcal{T}$ and the $G$-equivariant $K$-theory of the algebra $C_{c}^{\infty}(\mathcal{G})$. 
Proof. We have for any $\left(g_{1}, g_{2}\right) \in G_{1}^{2}$ :

$$
\begin{aligned}
\left\langle\tau_{C}^{g_{1} g_{2}}, e\right\rangle & =\left(\tau_{C}^{\mathcal{H}} \sharp \operatorname{Tr}\right)\left(e^{g_{1} g_{2}}, e, \cdots, e\right) \\
& =\left(\tau_{C}^{\mathcal{H}} \sharp \operatorname{Tr}\right)\left(\left(e^{g_{1}}\right)^{g_{2}}, e, \cdots, e\right) \\
& =\left(\tau_{C}^{\mathcal{H}} \sharp \operatorname{Tr}\right)\left(e^{g_{1}}, \cdots, e,{ }^{g_{2}} e\right) \\
& =\left(\tau_{C}^{\mathcal{H}} \sharp \operatorname{Tr}\right)\left(e, \cdots, e,{ }^{g_{1}}\left(e^{g_{2}}\right)\right) \\
& =\left(\tau_{C}^{\mathcal{H}} \sharp \operatorname{Tr}\right)\left(e, \cdots, e,\left(e^{g_{2}}\right)^{g_{1}}\right) \\
& =\left(\tau_{C}^{\mathcal{H}} \sharp \operatorname{Tr}\right)\left(e^{g_{2} g_{1}}, e, \cdots, e\right) .
\end{aligned}
$$

This is a consequence of Theorem 4 of the $G$-invariance of $e$ and of the cyclicity of the even cocycle $\tau_{C}^{\mathcal{H}}$. Hence we get

$$
\left\langle\tau_{C}^{g_{1} g_{2}}, e\right\rangle=\left\langle\tau_{C}^{g_{2} g_{1}}, e\right\rangle .
$$

Assume now that $u$ is a $G$-invariant invertible element of the algebra $\mathcal{A}:=$ $\left(C_{c}^{\infty}(\mathcal{G}) \oplus \mathbb{C}\right) \otimes \operatorname{End}(X)$ and let $\rho: \mathcal{A} \rightarrow \mathcal{A}$ be conjugation by $u$ in $\mathcal{A}$. By Lemma 3 below, we know that there exists a cyclic odd cochain $\varphi$ on $\mathcal{A}$ such that

$$
\rho^{*}\left(\tau_{C}^{\mathcal{H}} \sharp \operatorname{Tr}\right)-\left(\tau_{C}^{\mathcal{H}} \sharp \operatorname{Tr}\right)=b(\varphi),
$$

and which is, in addition, $G_{1}$-equivariant. Therefore, we deduce from the definition of the Hochschild coboundary $b$ and using that $e$ is $G$-invariant, the relation:

$$
b \varphi\left(e^{g}, e, \cdots, e\right)=\varphi\left(e^{g}, e, \cdots, e\right) .
$$

In other words, the terms in $b \varphi$ cancel each other out, except the last term. But

$$
\begin{aligned}
\varphi\left(e^{g}, e, \cdots, e\right) & =\varphi\left(e, \cdots, e,{ }^{g} e\right) \\
& =\varphi\left(e, \cdots, e, e^{g}\right) \\
& =-\varphi\left(e^{g}, e, \cdots, e\right) .
\end{aligned}
$$

Hence, we can deduce that $b \varphi\left(e^{g}, e, \cdots, e\right)=0$ and so that

$$
\left\langle\tau_{C}^{g}, e\right\rangle=\left\langle\tau_{C}^{g}, e^{\prime}\right\rangle, \quad \forall g \in G_{1} .
$$

Now by classical $K$-theory techniques, we can easily deduce from the above discussion that if $x=\left[e_{1}\right]-\left[e_{2}\right] \in K^{G}\left(C_{c}^{\infty}(\mathcal{G})\right)$, then the complex number

$$
\left(\tau_{C}^{g}\right)_{*}(x):=\left\langle\tau_{C}^{g}, e_{1}\right\rangle-\left\langle\tau_{C}^{g}, e_{2}\right\rangle
$$

only depends on the $G$-equivariant $K$-theory class $x$. We get in this way a group morphism:

$$
\left(\tau_{C}^{g}\right)_{*}: K^{G}\left(C_{c}^{\infty}(\mathcal{G})\right) \longrightarrow \mathbb{C} .
$$

Finally, we point out that the class $\psi_{\Theta}^{\mathcal{H}}\left(e^{g}(d e)^{2 k}\right)$ is closed for any idempotent $e$ and any $k \geq 0$. More precisely, define $U:=2 e-1$. Then $U^{2}=1$ and we have

$$
U\left(d e^{g}\right)=-\left(d e^{g}\right) U \quad \text { and } \quad U(d e)=-(d e) U .
$$

Therefore, and since $\psi_{\Theta}^{\mathcal{H}}$ is tracial, we get

$$
\begin{aligned}
\psi_{\Theta}^{\mathcal{H}}\left(d\left(e^{g}\right)(d e)^{2 k}\right) & =\psi_{\Theta}^{\mathcal{H}}\left(U^{2} d\left(e^{g}\right)(d e)^{2 k}\right) \\
& =-\psi_{\Theta}^{\mathcal{H}}\left(U d\left(e^{g}\right)(d e)^{2 k} U\right) \\
& =-\psi_{\Theta}^{\mathcal{H}}\left(U^{2} d\left(e^{g}\right)(d e)^{2 k}\right) \\
& =-\psi_{\Theta}^{\mathcal{H}}\left(d\left(e^{g}\right)(d e)^{2 k}\right) .
\end{aligned}
$$

If now $C=d C^{\prime}$ with $C^{\prime}$ a $\Gamma$-invariant current, then from the definition of the cyclic cocycle $\tau_{d C^{\prime}}^{\mathcal{H}}$, one thus deduces that $\left(\tau_{C}^{g}\right)_{*}(x)=0$. 
Remark 4. The pairing of Theorem 5 only depends on the equivariant cyclic cohomology class of $\tau_{C}^{\mathcal{H}}$, i.e., if we work with cyclic cochains $\varphi$ that are $G_{1}$-equivariant. Note that the Hochschild coboundary $b$ as well as the cyclic operator $B$, see [17, 30], preserve this equivariance property. We therefore have a well-defined subcomplex of the cyclic complex. Moreover, the periodic operator $S$ [17] also preserves this subcomplex and the above pairing is easily seen to depend only on the equivariant periodic cyclic cohomology class of $\tau_{C}^{\mathcal{H}}$.

Remark 5. In the above proof, we have used an equivariant cyclic theory. This theory is explicitly described in [9] with an explicit construction of the Chern-Connes character from equivariant $K$-theory to equivariant periodic homology, which implies the existence of the above pairing. Compare also with [28].

Lemma 3. Let $G$ be a compact Lie group and let $G_{1}$ be some subgroup of $G$. Let $\mathcal{A}$ be a unital $G$-algebra and let $u$ be an invertible $G$-invariant element of $\mathcal{A}$. Denote by $\rho$ the interior automorphism of $\mathcal{A}$ defined by $u$. Then for any cyclic cocycle $\tau$ on $\mathcal{A}$ that is $G_{1}$-equivariant, there exists a $G_{1}$-equivariant cyclic cochain $\varphi$ on $\mathcal{A}$ such that

$$
\rho^{*} \tau-\tau=b \varphi .
$$

Proof. This lemma is proved in the nonequivariant case by Connes in 17. One then only needs to check that the cyclic cochain $\varphi$ constructed by Connes is actually $G_{1}$-equivariant. This is easy and is proved in [9].

Corollary 1. Any closed $\Gamma$-invariant current on the manifold $\mathcal{T}$ induces a group morphism:

$$
\left(\tau_{C}^{[g]}\right)_{*}: K^{G}\left(C_{c}^{\infty}(\mathcal{G})\right)_{[g]} \rightarrow \mathbb{C},
$$

defined for any $x \in K^{G}\left(C_{c}^{\infty}(\mathcal{G})\right)$ and any $\chi \in R(G)$ with $\chi([g]) \neq 0$, by

$$
\left(\tau_{C}^{[g]}\right)_{*}\left(\frac{x}{\chi}\right):=\frac{\left(\tau_{C}^{[g]}\right)_{*}(x)}{\chi([g])} .
$$

Proof. If $E$ is a finite-dimensional representation of $G$ and if $x=[e]-\left[e^{\prime}\right] \in$ $K^{G}\left(C_{c}^{\infty}(\mathcal{G})\right)$, then the definition of the map $\tau_{C}^{g}$ gives

$$
\left\langle\tau_{C}^{g}, e \otimes E\right\rangle=\left\langle\tau_{C}^{g}, e\right\rangle \times \operatorname{Tr}\left(\left.g\right|_{E}\right) \quad \text { and } \quad\left\langle\tau_{C}^{g}, e^{\prime} \otimes E\right\rangle=\left\langle\tau_{C}^{g}, e^{\prime}\right\rangle \times \operatorname{Tr}\left(\left.g\right|_{E}\right) .
$$

Therefore, we have

$$
\left\langle\tau_{C}^{g}, x E\right\rangle=\left\langle\tau_{C}^{g}, x\right\rangle \times \operatorname{Tr}\left(\left.g\right|_{E}\right),
$$

which completes the proof.

\section{FiXed POINT FORMulae}

In this section, we shall investigate fixed point formulae for flat bundles. Our main tools will be the longitudinal index theorem in cyclic cohomology [32, 15] together with the longitudinal Lefschetz theorem in $K$-theory [6], see Section 1 .

We keep the notation of the previous sections. So $\Gamma$ is the fundamental group of the smooth compact manifold $M$, and we are given a smooth locally free action $\varrho$ of $\Gamma$ on a smooth compact manifold $\mathcal{T}$. The universal cover of $M$ is denoted as before by $\tilde{M}$. We are again interested in the foliation $F$ of the smooth compact manifold $V:=\tilde{M} \times_{\Gamma} \mathcal{T}$ whose leaves are the projections of $\tilde{M} \times\{t\}$ in $V$ when $t$ runs through $\mathcal{T}$. We assume again and from now on that the compact Lie group $G$ is topologically cyclic and generated by the leaf-preserving isometry $g$. The manifold $\tilde{M}$ is again 
assumed orientable and oriented for simplicity. The longitudinal vector bundle $F$ is then oriented by the induced orientation. We denote again by $\tilde{X}$ the smooth transverse submanifold of $\tilde{M} \times \mathcal{T}$ defined in Section 1 and whose projection in $V$ coincides with the fixed point submanifold $X=V^{G}$ of $G$. Since the cyclic index theorem for the compact foliated manifold $\left(V^{G}, F^{G}\right)$ will be used in the sequel, we proceed now to recall it for the convenience of the reader. The main references are [16, 15, 32, 27].

In order to get an explicit geometric computation of the index of a longitudinally elliptic pseudodifferential operator $P$ on $\left(X, F_{X}\right)$ by a universal formula, one uses the pairing of $K$-theory with cyclic cohomology and the more computable image of $\operatorname{Ind}(P)$ under the pairing with some cyclic cocycle on the algebra $C_{c}^{\infty}\left(\mathcal{G}_{X}\right)$. In the case of cyclic cocycles arising from $\Gamma$-invariant closed currents on $\mathcal{T}$ as studied in the previous sections, we get a cyclic index

$$
\operatorname{Ind}_{C}(P):=\left\langle\tau_{C}, \operatorname{Ind}(P)\right\rangle \quad \in \mathbb{C} .
$$

Let us first treat the case where $X=V$, say when the fixed point submanifold $V^{G}$ is obtained by suspension of an action of $\Gamma$. So, let $\mathcal{T} \times_{\Gamma} T^{*} \tilde{M}$ be the total space of the colongitudinal bundle of the foliation $(V, F)$. Since the pseudodifferential operator $P$ is elliptic, its principal symbol defines, by classical $K$-theory arguments, a class $[\sigma(P)]$ in the (compactly supported) $K$-theory group $K^{0}\left(\mathcal{T} \times_{\Gamma} T^{*} \tilde{M}\right)$ [4]. One then uses the Chern character Ch to send $[\sigma(P)]$ into a compactly supported rational cohomology class

$$
\operatorname{Ch}[\sigma(P)] \in \mathrm{H}_{c}^{2 *}\left(\mathcal{T} \times_{\Gamma} T^{*} \tilde{M}, \mathbb{Q}\right) .
$$

Integration along the fibres of the oriented fibration

$$
\pi_{F}: \mathcal{T} \times_{\Gamma} T^{*} \tilde{M} \rightarrow V=\mathcal{T} \times_{\Gamma} \tilde{M}
$$

then yields a cohomology class $\pi_{F} ! \mathrm{Ch}[\sigma(P)] \in \mathrm{H}^{*}(V, \mathbb{R})$, which is represented by a $\Gamma$-equivariant differential form on $\tilde{M} \times \mathcal{T}$. The cyclic index theorem then relates the analytic $C$-index $\operatorname{Ind}_{C}(P)$ of the operator $P$ defined above with some $C$-integral of the geometric class $\pi_{F}$ ! $\mathrm{Ch}[\sigma(P)]$. More precisely, let $\phi$ be the integration along the leaves constructed in Section 2 but with $\mathcal{H}$ being the vertical distribution of the fibration $p: V \rightarrow M$. Then we can state more precisely:

Theorem 6. 32, 15, 27 Under the above notation and for any even closed $\Gamma$ invariant current $C$ on $\mathcal{T}$, the following Atiyah-Singer formula holds:

$$
\operatorname{Ind}_{C}(P)= \pm\left\langle\phi\left(\pi_{F} ! \operatorname{Ch}[\sigma(P)] p^{*} \operatorname{Td}(M)\right), C\right\rangle,
$$

where $\operatorname{Td}(M)$ is the Todd class of the vector bundle $T M \otimes \mathbb{C}$ and $p: V \rightarrow M$ is the projection. The sign in the index formula depends on the normalization of the Chern character, see [4].

Note that $\pi_{F}$ ! $\mathrm{Ch}[\sigma(P)] p^{*} \operatorname{Td}(M)$ is a cohomology class of a $\Gamma$-equivariant differential form on $\tilde{M} \times \mathcal{T}$ so that the RHS in the index formula makes sense. One can

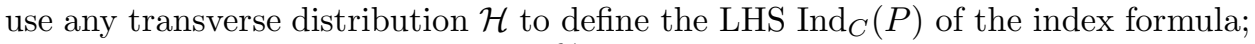
only the class of the cyclic cocycle $\tau_{C}^{\mathcal{H}}$ in cyclic cohomology is really involved.

We shall need a slight modification of the index formula of Theorem 6 More precisely, we need to deal with the closed submanifold $X=V^{G}$ of $V$, which does not arise necessarily from a $\Gamma$ suspension as before. Let $\tilde{X}$ be the smooth closed submanifold of $\tilde{M} \times \mathcal{T}$ defined in Section 1 and which is everywhere transverse to the leaves $\tilde{M} \times\{t\}$ and globally $\Gamma$-invariant. 
A smooth differential form $\omega$ on $X$ can then be viewed as a $\Gamma$-invariant differential form $\tilde{\omega}$ on $\tilde{X}$. The construction of the integration along the leaves $\phi$ on the flat bundle $V$ can be slightly modified to deal with our compact foliated manifold $\left(X, F_{X}\right)$.

For any $t \in A^{G}$, let $\Gamma_{1, t}$ be the subgroup of $\Gamma$ of those elements $\gamma \in \Gamma$ that preserve the leaf submanifold $(\tilde{M} \times\{t\}) \cap \tilde{X}$. Choose a fundamental domain $U_{t}$ for the free and proper action of $\Gamma_{1, t}$ on this leaf. Now we define, for any $t \in A^{G}$,

$$
\phi_{U}^{X}(\tilde{\omega})(t):=\int_{U_{t}} \tilde{\omega}(t, \tilde{m}) d \tilde{m},
$$

and denote by $\phi^{X}(\tilde{\omega})$ the class thus obtained in the homology group $\mathrm{H}_{0}\left(\Gamma, \Omega^{*}\left(A^{G}\right)\right)$. This class does not depend on the particular choice of the fundamental domains $U_{t}$. So if we denote by $\Omega^{*}(\tilde{X})^{\Gamma}$ the space of $\Gamma$-invariant differential forms on $\tilde{X}$, we get a well-defined Haefliger map [23]:

$$
\phi^{X}: \Omega^{*}(\tilde{X})^{\Gamma} \longrightarrow \mathrm{H}_{0}\left(\Gamma, \Omega^{*}\left(A^{G}\right)\right), \quad \omega \longmapsto \phi^{X}(\omega) .
$$

If now $C$ is a closed even $\Gamma$-invariant current on $\mathcal{T}$, then $C$ restricts to differential forms that are supported inside the open submanifold $A^{G}$ and yields a closed $\Gamma$ invariant current on the smooth compact manifold $A^{G}$. The integral of $\phi^{X}(\omega)$ against $C$ thus yields a complex number $\left\langle\phi^{X}(\omega), C\right\rangle$.

The Chern character of the class $[\sigma(P)]$ is then a compactly supported cohomology class $\mathrm{Ch}[\sigma(P)]$, which can be viewed as the class of a $\Gamma$-invariant differential form on $F_{\tilde{X}}^{*}$. The index theorem for the compact foliated manifold $\left(X, F_{X}\right)$ can then be stated as follows:

Theorem 7. Let $P$ be a longitudinally elliptic pseudodifferential operator on $\left(X, F_{X}\right)$ with coefficients in a vector bundle $E$. Let $C$ be a $\Gamma$-invariant closed current on $\mathcal{T}$. Then the $C$-index $\operatorname{Ind}_{C}(P):=\left\langle\tau_{C}^{X}, \operatorname{Ind}(P)\right\rangle$ of $P$ can be computed by the following formula:

$$
\operatorname{Ind}_{C}(P)= \pm\left\langle\phi^{X}\left(\pi_{F_{X}} ! \operatorname{Ch}[\sigma(P)] p^{*} \operatorname{Td}\left(p_{0}\left(B^{G}\right)\right),\left.C\right|_{A^{G}}\right\rangle .\right.
$$

This theorem is proved in [15] in a more general setting. The methods used in 27. also enable us to deduce it.

We now introduce again the action of the topologically cyclic compact Lie group $G$ on our foliated flat bundle $V \rightarrow M$. We assume as in Section 3 that the dense subgroup $G_{1}$ of $G$ generated by $g$ acts on $V$ by leaf-preserving isometries and that $g$ is induced by an isometry $\tilde{g}$ for some $\Gamma$-invariant metric of $\tilde{M} \times \mathcal{T}$.

Definition 4. Let $P$ be a $G$-invariant longitudinally elliptic pseudodifferential operator on $(V, F ; E)$ and let $C$ be a closed $\Gamma$-invariant current on $\mathcal{T}$. Then the Lefschetz number $\mathbb{L}_{C}(g ; P)$ of $g$ with respect to $P$ is defined as the image under the morphism $\left(\tau_{C}^{g}\right)_{*}: K^{G}\left(C_{c}^{\infty}(\mathcal{G})\right) \rightarrow \mathbb{C}$ of the $G$-index of $P$. So we have

$$
\mathbb{L}_{C}(g ; P):=\left\langle\left(\tau_{C}^{g}\right)_{*}, \operatorname{Ind}_{G}(P)\right\rangle .
$$

The Lefschetz $C$-number is intimately related with the fixed point submanifold $V^{G}$ of $g$. More precisely, the pairing defined in Section 3 between localized $G$ equivariant $K$-theory and invariant homology enables us to view $\mathbb{L}_{C}(g ; P)$ as the image under the map induced by $\tau_{C}^{g}$, of the Lefschetz class $\mathbb{L}(g ; P)$. On the other hand, a necessary condition that the number $\mathbb{L}_{C}(g ; P)$ be nontrivial is that the 
solutions of $P_{t}$ are nontrivial for a $C$-nontrivial subset of $\mathcal{T}$. Therefore, this number carries precious analytic information that we shall compute in topological terms.

We keep the notation of Section[1. The equivariant class of the principal symbol of $P$ can then be restricted to a class $i^{*}[\sigma(P)] \in K_{G}\left(F^{G, *}\right)$. We can define evaluation $x \mapsto x(g)$ at $g$ for any $x \in K_{G}\left(F^{G, *}\right) \simeq K\left(F^{G, *}\right) \otimes R(G)$ by using the evaluation of the character components. For $x \in K_{G}\left(F^{G, *}\right)$, the evaluation $x(g)$ of $x$ at $g$ thus belongs to the group $K\left(F^{G, *}\right) \otimes \mathbb{C}$.

Theorem 8. The C-Lefschetz number of the isometry $g$ with respect to the longitudinally elliptic pseudodifferential $G$-invariant operator $P$ is given by the following fixed point topological formula:

$$
\mathbb{L}_{C}(g ; P)=\left\langle\phi^{V^{G}}\left(\pi_{F^{G}} ! \frac{\mathrm{Ch}\left(i^{*}[\sigma(P)]\right)(g)}{\operatorname{Ch}\left(\lambda_{-1}\left(N^{G} \otimes \mathbb{C}\right)(g)\right)} p^{*} \operatorname{Td}\left(p_{0}\left(B^{G}\right)\right)\right),\left.C\right|_{A^{G}}\right\rangle .
$$

Proof. We apply the Lefschetz theorem in the $g$-localized $G$-equivariant $K$-theory recalled in Section 1 We obtain

$$
\mathbb{L}(g ; P)=\left(\operatorname{Ind} \otimes R(G)_{g}\right)\left(\frac{i^{*}[\sigma(P)]}{\lambda_{-1}\left(N^{G} \otimes \mathbb{C}\right)}\right) \quad \in K^{G}\left(C_{c}^{\infty}(\mathcal{G})\right)_{g} .
$$

Therefore, if we apply the additive map $\left(\tau_{C}^{g}\right)_{*}$ to this equality, we get

$$
\mathbb{L}_{C}(g ; P)=\left[\left(\tau_{C}^{g}\right)_{*} \circ\left(\operatorname{Ind} \otimes R(G)_{g}\right)\right]\left(\frac{i^{*}[\sigma(P)]}{\lambda_{-1}\left(N^{G} \otimes \mathbb{C}\right)}\right) .
$$

But an easy inspection of the map $\left(\tau_{C}^{g}\right)_{*}$ shows that it coincides, in restriction to the elements of $K^{G}\left(C_{c}^{\infty}\left(\mathcal{G}_{X}\right)\right)_{g}$, and according to the isomorphism

$$
K^{G}\left(C_{c}^{\infty}\left(\mathcal{G}_{X}\right)\right)_{g} \simeq K\left(C_{c}^{\infty}\left(\mathcal{G}_{X}\right)\right) \otimes R(G)_{g},
$$

with the map $\left(\tau_{C}\right)_{*} \otimes e v_{g}$, where $\tau_{C}=\tau_{C}^{1}$ and $e v_{g}: R(G)_{g} \rightarrow \mathbb{C}$ is evaluation at $g$. Thus we get

Finally, we point out that

$$
\mathbb{L}_{C}(g ; P)=\left(\operatorname{Ind}_{C} \otimes e v_{g}\right)\left(\frac{i^{*}[\sigma(P)]}{\lambda_{-1}\left(N^{G} \otimes \mathbb{C}\right)}\right) .
$$

$$
\left(\operatorname{Ind}_{C} \otimes e v_{g}\right)(x)=\left(\operatorname{Ind}_{C} \otimes \mathbb{C}\right)(x(g)), \quad \forall x \in K^{G}\left(C_{c}^{\infty}\left(\mathcal{G}_{X}\right)\right)_{g} .
$$

To end the proof we simply use Theorem 7 .

Many applications of the cyclic Lefschetz theorem for foliated bundles can be deduced by immediate generalization of classical ones; see, for instance, [5, 4, 2, 24, 25. When the $\Gamma$-invariant current $C$ is, for instance, a $\Gamma$-invariant positive measure $\mu$, we obtain as a byproduct the results of 24 .

In the case of invariant measures, all the results of the present paper can be generalized to the case where $\mathcal{T}$ is not necessarily a smooth manifold, and is now only a compact topological space on the one hand and to the case where the fixed point submanifold is not necessarily transverse to the foliation on the other hand. The definition of the normal vector bundle has then to be appropriately modified. Then the action $\varrho$ is by homeomorphisms and the measure $\mu$ of interest is any positive measure that is invariant under the action $\varrho$ of $\Gamma$. The characteristic classes that appear then in the Lefschetz formula must be replaced by longitudinal ones as in [10]. There is an important application of this situation which concerns the study of quasi-crystals à la Bellissard, where the space $\mathcal{T}$ is a Cantor space. The image of the $K$-theory of the $C^{*}$-algebra of the lamination $(V, F)$ under the 
trace (or 0-cocycle) $\tau_{\mu}$ associated with $\mu$ has been computed in [10], answering a conjecture of J. Bellissard. The result is

$$
\left(\tau_{\mu}\right)_{*}\left(K\left(C^{*}(V, F)\right)=\mu(C(\mathcal{T}, \mathbb{Z}))\right.
$$

where $C(\mathcal{T}, \mathbb{Z})$ is the space of continuous integer-valued functions on the Cantor space $\mathcal{T}$. As an easy consequence of this computation, one can deduce that the characteristic numbers appearing in the RHS of the measured Lefschetz formula belong to the subgroup $R(G)(g) \otimes \mu(C(\mathcal{T}, \mathbb{Z}))$ of complex numbers; compare with the example treated in 24]. So when $g$ is an involution, we deduce that such numbers must belong to $\mu(C(\mathcal{T}, \mathbb{Z}))$.

Another interesting invariant current appears when one works with the $\Gamma$-invariant index theory of $\mathcal{T}$ as in [18] 19]. So assume, for instance, that the group $\Gamma$ acts by orientation-preserving isometries of the smooth compact oriented evendimensional manifold $\mathcal{T}$. Denote by $\mathcal{L}\left(A^{G}\right)$ the Hirzebruch $\mathcal{L}$-polynomial in the Pontrjagin classes of the oriented compact submanifold $A^{G}$ of $\mathcal{T}$ [4]. Then the characteristic number

$$
\int_{A^{G}} \phi^{V^{G}}\left(\pi_{F^{G}} ! \frac{\mathrm{Ch}\left(i^{*}[\sigma(P)]\right)(g)}{\operatorname{Ch}\left(\lambda_{-1}\left(N^{G} \otimes \mathbb{C}\right)(g)\right)} p^{*} \operatorname{Td}\left(p_{0}\left(B^{G}\right)\right)\right) \mathcal{L}\left(A^{G}\right)
$$

belongs to the subgroup $R(G)(g)$ of complex numbers. In particular, for involutions, we get an integer.

\section{REFERENCES}

[1] M. F. Atiyah, Elliptic operators, discrete groups and von Neumann algebras, Colloque "Analyse et Topologie" en l'Honneur de Henri Cartan (Orsay, 1974), pp. 43-72. Asterisque, No. 32-33, Soc. Math. France, Paris, 1976. MR 54:8741]

[2] M. F. Atiyah and F. Hirzebruch, Spin-manifolds and group actions, 1970 Essays on Topology and Related Topics (Mémoires dédiés à Georges de Rham) pp. 18-28, Springer-Verlag, New York. MR 43:4064

[3] M. F. Atiyah, V. K. Patodi and I. M. Singer, Spectral asymmetry and Riemannian geometry. III, Math. Proc. Cambridge Philos. Soc. 79 (1976), no. 1, 71-99. MR 53:1655c

[4] M. F. Atiyah and I. M. Singer, The index of elliptic operators. III, Ann. of Math. (2) 87 (1968), 546-604. MR 38:5245

[5] M. F. Atiyah and G. Segal, The index of elliptic operators. II, Ann. of Math. (2) 87 (1968) 531-545. MR 38:5244

[6] M.-T. Benameur, A longitudinal Lefschetz theorem in K-theory, K-Theory 12 (1997), no. 3, 227-257. MR 98j:19007

[7] M.-T. Benameur, Cyclic cohomology and the family Lefschetz theorem, to appear in Math. Ann.

[8] M.-T. Benameur and V. Nistor, Homology of complete symbols and noncommutative geometry, "Collected papers on Quantization of Singular Symplectic Quotients", Progress in Math. series of Birkhäuser 198, (2001).

[9] M.-T. Benameur, On the equivariant Chern-Connes character in noncommutative geometry, preprint.

[10] M.-T. Benameur and H. Oyono-Oyono, Computation of the range of the trace for quasicrystals, preprint.

[11] J.-M. Bismut and J. Cheeger, Families index for manifolds with boundary, superconnections, and cones, I. Families of manifolds with boundary and Dirac operators, J. Funct. Anal. 89 (1990), no. 2, 313-363. MR 91e:58180

[12] A. V. Brenner, M. A. Shubin, The Atiyah-Bott-Lefschetz formula for elliptic complexes on a manifold with boundary, (Russian) Translated in J. Soviet Math. 64 (1993), no. 4, 1069-1111. Itogi Nauki i Tekhniki, Current problems in mathematics. Newest results, Vol. 38 (Russian), 119-183, 186, Akad. Nauk SSSR, Vsesoyuz. Inst. Nauchn. i Tekhn. Inform., Moscow, 1990. MR 93k:58212 
[13] A. Connes, A survey of foliations and operator algebras, Proc. Sympos. Pure Math. 38, Amer. Math. Soc., Providence, RI, 1982. MR 84m:58140

[14] A. Connes. Sur la théorie non commutative de l'integration (French), Algèbres d'opérateurs (Sém., Les Plans-sur-Bex, 1978), pp. 19-143, Lecture Notes in Math., 725, Springer-Verlag, Berlin, 1979. MR 81g:46090

[15] A. Connes, cours du collège de France, 94/95.

[16] A. Connes, Noncommutative Geometry, Academic Press, San Diego, CA, 1994. MR 95j:46063

[17] A. Connes, Noncommutative differential geometry, Inst. Hautes Études Sci. Publ. Math. 62 (1985), 257-360. MR 87i:58162

[18] A. Connes and H. Moscovici, The local index formula in noncommutative geometry, Geom. Funct. Anal. 5 (1995), no. 2, 174-243. MR 96e:58149]

[19] A. Connes and H. Moscovici, Hopf algebras, cyclic cohomology and the transverse index theorem, Comm. Math. Phys. 198 (1998), no. 1, 199-246. MR 99m:58186

[20] A. Connes and G. Skandalis, The longitudinal index theorem for foliations, Publ. Res. Inst. Math. Sci. 20 (1984), no. 6, 1139-1183. MR 87h:58209

[21] M. Crainic and I. Moerdijk, Foliation groupoids and their cyclic homology, Adv. Math. 157 (2001), no. 2, 177-197. MR 2002a:22004

[22] R. Douglas, S. Hurder and J. Kaminker, Cyclic cocycles, renormalization and eta-invariants, Invent. Math. 103 (1991), no. 1, 101-179. MR 91m:58152

[23] A. Haefliger, Some remarks on foliations with minimal leaves, J. Differential Geom. 15 (1980), 269-284. MR 82j:57027

[24] J. L. Heitsch and C. Lazarov, A Lefschetz theorem for foliated manifolds, Topology 29 (1990), no. 2, 127-162. MR 91g:58281

[25] J. L. Heitsch and C. Lazarov, Rigidity theorems for foliations by surfaces and spin manifolds, Michigan Math. J. 38 (1991), no. 2, 285-297. MR 92b:58220

[26] J. L. Heitsch and C. Lazarov, A general families index theorem, $K$-Theory 18 (1999), no. 2, 181-202. MR 2000h:58046

[27] X. Jiang, An index theorem on foliated flat bundles, K-Theory 12 (1997), no. 4, 319-359. MR 99f: 58197

[28] S. Klimek, W. Kondracki and A. Lesniewski, Equivariant entire cyclic cohomology. I. Finite groups, $K$-Theory 4 (1991), no. 3, 201-218. MR 92g:46091

[29] R. Lauter, B. Monthubert, and V. Nistor, Pseudodifferential operators on continuous family groupoids, Doc. Math. (electronic) 5 (2000) 625-655.

[30] J.-L. Loday, Cyclic homology. Appendix E by Maria O. Ronco. Grundlehren der Mathematischen Wissenschaften [Fundamental Principles of Mathematical Sciences], 301, SpringerVerlag, Berlin, 1992. MR 94a:19004

[31] R. Melrose and V. Nistor, Homology of pseudodifferential operators I. Manifolds with boundary, to appear in Amer. J. Math.

[32] V. Nistor, The index of operators on foliated bundles, J. Funct. Anal. 141 (1996), no. 2, 421-434. MR 97j:19005

[33] V. Nistor, A. Weinstein and Ping Xu, Pseudodifferential operators on differential groupoids, Pacific J. Math. 189 (1999), no. 1, 117-152. MR 2000c:58036

[34] P. Tondeur, Geometry of foliations, Monographs in Mathematics, 90. Birkhäuser-Verlag, Basel, 1997. MR 98d:53037

[35] E. Witten, The index of the Dirac operator in loop space, Elliptic curves and modular forms in algebraic topology (Princeton, NJ, 1986), 161-181, Lecture Notes in Math. 1326, SpringerVerlag, Berlin, 1988.

Institut Girard Desargues, Université Claude Bernard, Lyon 1, France

E-mail address: benameur@igd.univ-lyon1.fr 